\title{
FIRST CATALOG OF STRONG LENS CANDIDATES IN THE COSMOS FIELD
}

\author{
Cecile Faure, ${ }^{1,2}$ Jean-Paul Kneib, ${ }^{3,4}$ Giovanni Covone, ${ }^{3,5}$ Lidia Tasca, ${ }^{3}$ Alexie Leauthaud, ${ }^{3}$ Peter Capak, ${ }^{4}$ \\ Knud Jahnke, ${ }^{6}$ Vernesa Smolcic, ${ }^{6}$ Sylvain de la Torre, ${ }^{3}$ Richard Ellis, ${ }^{4}$ Alexis Finoguenov, ${ }^{7}$ \\ Anton Koekemoer, ${ }^{8}$ Oliver Le Fevre, ${ }^{3}$ Richard Massey, ${ }^{4}$ Yannick Mellier,, 9 \\ Alexandre Refregier, ${ }^{10}$ Jason Rhodes, ${ }^{4}$ Nick Scoville, ${ }^{4}$ Eva Schinnerer, ${ }^{6}$

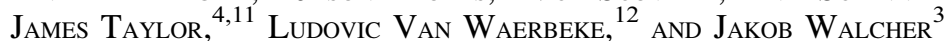 \\ Received 2007 March 5; accepted 2007 November 2
}

\begin{abstract}
We present the first catalog of 67 strong galaxy-galaxy lens candidates discovered in the $1.64 \mathrm{deg}^{2}$ Hubble Space Telescope COSMOS survey. Twenty of these systems display multiple images or strongly curved large arcs. Our initial search is performed by visual inspection of the data and is restricted, for practical considerations, to massive early-type lens galaxies with arcs found at radii smaller than $\sim 5^{\prime \prime}$. Simple mass models are constructed for the best lens candidates, and our results are compared to the strong lensing catalogs of the SLACS survey and the CASTLES database. These new strong galaxy-galaxy lensing systems constitute a valuable sample to study the mass distribution of early-type galaxies and their associated dark matter halos. We further expect this sample to play an important role in the testing of software algorithms designed to automatically search for strong gravitational lenses. From our analysis a robust lower limit is derived for the expected occurrence of strong galaxy-galaxy systems in current and future space-based wide-field imaging surveys. We expect that such surveys should uncover a large number of strong lensing systems (more than 10 systems per square degree), which will allow for a detailed statistical analysis of galaxy properties and will likely lead to constraints on models of gravitational structure formation and cosmology.
\end{abstract}

Subject headings: catalogs — galaxies: statistics — gravitational lensing

Online material: color figures

\section{INTRODUCTION}

Gravitational lensing is one of the most promising tools in modern astrophysics for probing the matter content of the universe directly and thereby constraining cosmological models. Applications of strong gravitational lensing include measurements of the Hubble constant using lensed quasars with time delays (Refsdal 1964; for time delay measurements see, e.g., Barkana 1997; Cohen et al. 2000; Burud et al. 2000; Burud et al. 2002a, 2002b; Fassnacht et al. 2002; Hjorth et al. 2002; Ofek \& Maoz 2003; Jakobsson et al. 2005; Kochanek et al. 2006; Fohlmeister et al. 2006; Vuissoz et al. 2007) and the determination of galaxy

1 Astronomisches Rechen-Institut für Astronomie der Universitat at Heidelberg, Mönchhofstrasse 12-14, 69120 Heidelberg, Germany.

2 Departamento de Astronomía, Pontificia Universidad Católica, Av Vicuña Mackenna 4860, 782-0486 Macul, Santiago, Chile.

3 Laboratoire d'Astrophysique de Marseille, UMR6110, CNRS-Université de Provence, BP 8, F-13376 Marseille Cedex 12, France.

${ }^{4}$ California Institute of Technology, MC 105-24, 1200 East California Boulevard, Pasadena, CA 91125.

5 Universitá Federico II, Department of Physical Sciences, via Cinthia 9, 80126, Naples, Italy.

${ }^{6}$ Max-Planck-Institut für Astronomie, Königstuhl 17, 69117 Heidelberg, Germany.

${ }^{7}$ Max-Planck-Institut für extraterrestrische Physik, Giessenbachstrasse, 85748, Garching, Germany.

${ }^{8}$ Space Telescope Science Institute, 3700 San Martin Drive, Baltimore, MD 21218.

9 Institut d'Astrophysique de Paris, UMR 7095, 98 bis Boulevard Arago, 75014 Paris, France.

${ }^{10}$ Service d'Astrophysique, CEA/Saclay, 91191 Gif-sur- Yvette, France.

11 Department of Physics and Astronomy, University of Waterloo, Waterloo, ON N2L 3G1, Canada.

12 Department of Physics and Astronomy, University of British Columbia, 6224 Agricultural Road, Vancouver, BC V6T 1Z1, Canada. and cluster mass distributions using multiple images (e.g., Guzik \& Seljak 2002; Mandelbaum et al. 2006; Read et al. 2007). Moreover, homogeneous samples of strong lenses allow for a statistical determination of the properties of lensing galaxies (e.g., Kochanek 1996; Rusin \& Ma 2001; Chae et al. 2006; Chae 2007), and samples of strong lenses selected based on source properties are used to test cosmological models (e.g., Fukugita \& Turner 1991; Wambsganss et al. 2005; Moeller et al. 2007; Hilbert et al. 2007).

The giant arcs that are observed around massive clusters of galaxies are among the most spectacular lensing phenomena (e.g., Gladders et al. [2002] searched giant arcs in the Red-Sequence Cluster Survey [RCS]; Scarpine et al. [2006], Hennawi et al. [2008], and Estrada et al. [2007] searched in the Sloan Digital Survey [SDSS]). These cases are relatively rare because each square degree of sky contains only about one cluster of sufficient mass to produce a giant arc. Much more common are the strong lensing events found around massive early-type galaxies. The first studies of galaxy-galaxy strong lensing systems targeted distant, bright lensed sources, including quasars (ESO Hamburg survey: Wisotzki et al. 1996; CfA-Arizona-ST-LEns-Survey: Peng et al. 1997, Muñoz et al. 1998) and radio sources (Jodrell Bank-VLA Astrometric survey: Patnaik et al. 1992, Browne et al. 1998; Cosmic Lens All Sky Survey: Jackson et al. 1995, Myers et al. 1995). The identification of multiple images in these systems is generally unambiguous and does not require very deep observations but requires imaging over a large fraction of the sky.

The advent of deep and wide optical imaging combined with large spectroscopic surveys has greatly broadened the opportunities and means by which strong galaxy-galaxy lensing events can be found. Indeed, since we expect that $\sim 0.1 \%$ of massive galaxies (the luminous elliptical galaxies) strongly distort and magnify a galaxy source at $z>1$ (Miralda-Escudé \& Léhar 1992), new 
wide-field surveys are likely to discover many hundreds of fainter galaxy-galaxy strong lensing systems. Spectroscopic identification of two galaxies at different redshifts in the same SDSS fiber has proved to be a successful method in the Sloan Lens ACS survey (SLACS; Bolton et al. 2006). Deep, wide-field imaging surveys (especially those utilizing the exquisite image resolution available from space) provide another avenue for locating strong galaxy-galaxy lenses. This was first attempted in the Hubble Space Telescope Medium Deep Survey (Griffith et al. 1994; Ratnatunga et al. 1999) and more recently in the Great Observatories Origins Deep Survey field (GOODS; Dickinson et al. 2001; Fassnacht et al. 2004) or in the AEGIS survey (Moustakas et al. 2007). Automated software for identifying strong lenses in HST image archives are currently under development (Marshall et al. 2005; Moustakas et al. 2007) and appear to be very promising. Searches for strong lens systems are also underway from groundbased wide-field surveys. In particular, the SL2S initiative (Cabanac et al. 2007) was able to find a few lenses per square degree in the Canada-France-Hawaii Telescope (CFHT) LS wide survey. However, ground-based imaging surveys are necessarily limited to finding systems with large deflection angles because of the seeing limitation compared to space-based observations.

The $1.64 \mathrm{deg}^{2}$ Hubble Space Telescope COSMOS survey (Scoville et al. 2007) provides an excellent opportunity to locate and study a large number of strong galaxy-galaxy lensing systems. It includes the largest contiguous high-resolution astronomical imaging survey ever performed from space. Although observations with the HST Advanced Camera for Surveys (ACS) have only been obtained in a single band (F814W filter), multiwavelength coverage of this equatorial field is provided in the optical band by deep multicolor imaging from Suprime on the Subaru telescope and Megacam at CFHT.

In this paper, we present the first systematic attempt to locate strong galaxy-galaxy lensing systems in the COSMOS field. The data set used for our investigation is described in $\S 2$, and the techniques used to uncover strong galaxy-galaxy lensing candidates are discussed in $\S 3$. Our findings are presented in $\S 4$. The lens potential modeling scheme is discussed in $\S 5$, and we compare our lens sample to other strong lens catalogs in $\S 6$. Finally, the results are discussed in $\S 7$. Throughout this paper, galaxy magnitudes are quoted in the $\mathrm{AB}$ system, and cosmological distances are calculated assuming a $\Lambda$ CDM model with parameters $H_{0}=$ $73 \mathrm{~km} \mathrm{~s}^{-1} \mathrm{Mpc}^{-1}, \Omega_{m}=0.3$, and $\Omega_{\lambda}=0.7$.

\section{DATA}

To conduct our strong galaxy-galaxy lens search we take advantage of three key data sets of the COSMOS survey: the HST ACS high-resolution imaging, the Subaru Suprime imaging, and the CFHT Megacam multicolor imaging.

\subsection{HST Data}

We have analyzed the full ACS field of view of the COSMOS field that corresponds to $1.64 \mathrm{deg}^{2}$ observed during Cycles 12 and 13. We use data release version 1.2 of the COSMOS ACS data, which can be accessed through the dedicated database at IRSA. ${ }^{13}$ Individual exposures were combined using the MultiDrizzle software (Koekemoer et al. 2007) onto an output grid of pixel size $0.03^{\prime \prime}$. The limiting magnitude is about $I_{\mathrm{F} 814 \mathrm{~W}}=26.5 \mathrm{mag}$. A detailed description of the data is available in Scoville et al. (2007), and a detailed description of the ACS-selected galaxy catalog can be found in Leauthaud et al. (2007).

13 See http://irsa.ipac.caltech.edu/data/COSMOS/images/.

\subsection{Subaru and CFHT Data}

Multicolor imaging of the COSMOS field was obtained from the Subaru telescope with the Suprime camera (Miyazaki et al. 2002) in $B_{j}, V_{j}, g^{+}, r^{+}, i^{+}$, and $z^{+}$bands, and from the CFHT with the Megacam camera (Boulade et al. 2003) in the $u^{*}$ and $i^{*}$ bands. In these data, the seeing is generally better than $1^{\prime \prime}$. A photometric catalog was derived from a combination of these data, based on detections in the Subaru $i+$ band (Capak et al. 2007).

\subsection{Photometric Redshifts}

The publicly available Le Phare photometric redshift estimation code (Ilbert et al. 2006) has been used to measure the redshifts of 278,526 galaxies with $I_{\mathrm{F} 814 \mathrm{~W}}<25$ mag. Details concerning the multiwavelength photometry can be found in Mobasher et al. (2007). The $I_{\mathrm{F} 814 \mathrm{~W}}<25 \mathrm{mag}$ limit ensures good accuracy of both the photometric redshift estimation and the fit to the spectral energy distribution (SED). The ground-based photometric zero points were calibrated (and the SED templates adjusted accordingly) using 1095 spectroscopic redshifts from the zCOSMOS Survey (Lilly et al. 2006). Using eight bands, this method achieves a photometric redshift recovery accuracy of $\sigma_{\Delta z} /\left(1+z_{s}\right)=0.031$ with $\eta=1.0 \%$ of catastrophic errors, defined as $\Delta z /\left(1+z_{S}\right)>$ 0.15 (further details can be found in Mobasher et al. 2007).

\section{METHODOLOGY}

A four-step procedure is employed in order to identify strong galaxy-galaxy lens systems. In order, the four steps are the following:

1. Select a list of potential lenses from the photometric redshift catalog (hereafter the "P" catalog). ${ }^{14}$

2. Visually inspect the ACS images of all galaxies in the P catalog to produce a catalog of potential strong galaxy-galaxy lens systems (hereafter the "E" catalog; ${ }^{15}$ only 302 galaxies are in this catalog, the 35 others are missing from our personal archives).

3. Investigate the E catalog using multicolor images to check for color differences between the main galaxy and the potentially lensed object.

4. Subtract a galaxy surface brightness model of the foreground galaxy to determine the morphology and lensing configuration of the potentially lensed background galaxy.

In our methodology, systems that pass both steps 3 and 4 qualify as strong galaxy-galaxy lens candidates. We will now describe each step in more detail.

\subsection{The Potential Lens Catalog}

The ACS-based photometric redshift catalog limited to F814W = 25 mag contains 278,819 entries: it is unrealistic to visually inspect all galaxies in this catalog. Furthermore, this would be inefficient, because we know that a galaxy needs to be massive and not too distant to efficiently distort and strongly magnify a more distant galaxy. We therefore limit our search to intrinsically bright galaxies at moderate redshifts. Furthermore, for this first investigation of the COSMOS field, we only look at early-type galaxies (classified spectroscopically by the photometric redshift code), which generally have a simple, smooth surface brightness distribution. Once again, we emphasize that the main reason for

\footnotetext{
14 This catalog can be found at http://www.ari.uni-heidelberg.de/mitarbeiter/ cfaure/downloads/Pcatalog.cat.

${ }^{15}$ This catalog can be found at http://www.ari.uni-heidelberg.de/mitarbeiter/ cfaure/downloads/Ecatalog.cat.
} 


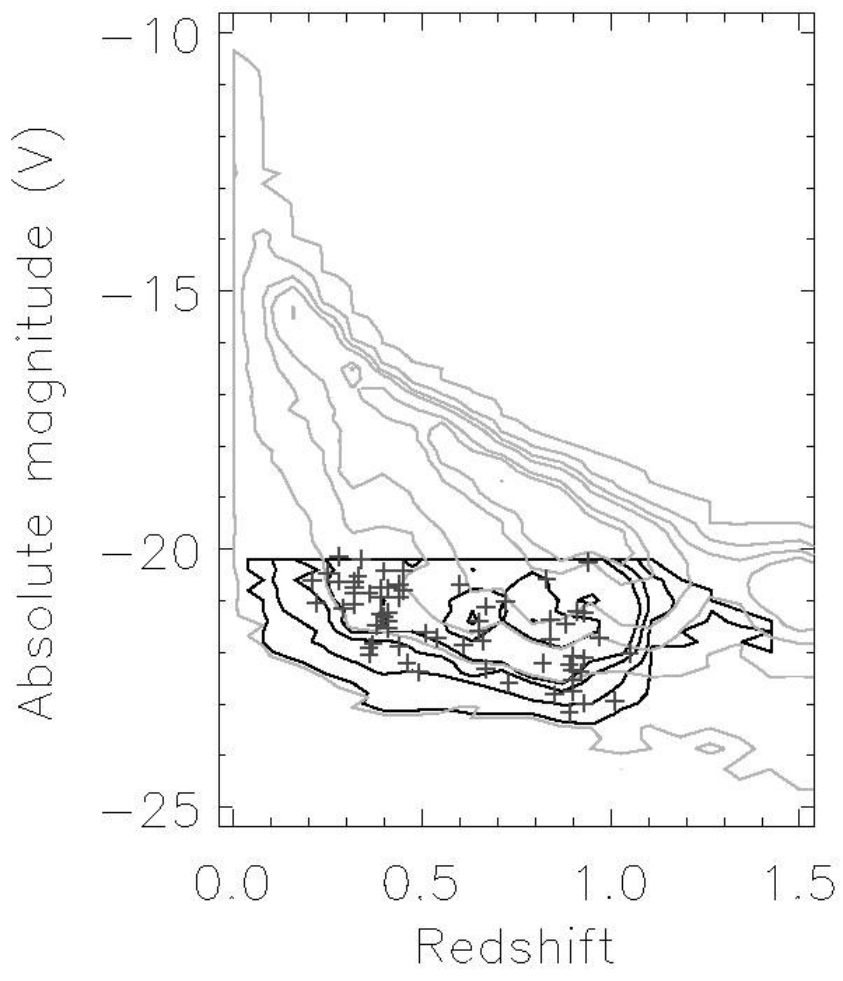

FIG. 1.-Galaxies of the COSMOS field: $V_{j}$-band absolute magnitude vs. photometric redshift. The gray solid contours show isodensity numbers of all the galaxies in the entire photometric catalog (levels: 1, 100, 300, 500, 1000, 1200, and 1600 galaxies). The black solid contours draw the isodensity numbers of galaxies in the $P$ catalog (levels: $1,10,30,50,100$, and 140 galaxies). The plus signs represent the lensing galaxy candidates described in $\S 4$. [See the electronic edition of the Supplement for a color version of this figure.] applying these selection criteria to the photometric redshift catalog is to reduce the sample of galaxies that need to be visually inspected. It is highly possible that additional strong lens systems will be found around galaxies that do not belong to our search sample. The number of strong lensing systems that we find is thus a lower limit to the total number of strong lensing systems in the COSMOS field.

To search for potential lenses, we select galaxies according to the following criteria:

1. Their photometric redshift must be in the range $0.2 \leq$ $z_{\text {phot }} \leq 1.0$. The lower redshift bound of $z=0.2$ is chosen to ensure that galaxies will indeed produce lensing events, and the upper bound is essentially motivated to ensure a good accuracy on the photometric redshift given that no deep infrared data are yet available for the COSMOS field. The galaxy is retained if the error bars at $68 \%$ confidence level overlap, even partially, the redshift range $[0.2 ; 1.0]$.

2. Their luminosity must be $M_{V}<-20 \mathrm{mag}$, on the assumption that the brightest galaxies are also likely to be the most massive ones with the largest cross section for lensing.

3. We limit our search to early-type galaxies that have been spectrally classified as such by the SED fitting of our photometric redshift algorithm. The motivation for this criterion is to focus on massive systems with a simple light distribution that is easy to fit and to subtract.

We obtain a final P catalog of 9452 bright elliptical galaxies (see Fig. 1). Further strong lensing searches using automated software will certainly relax these criteria.

During the course of our visual inspection, we serendipitously found some additional strong lens systems in which the lensing galaxy did not comply with the above limits. Although these constitute an inhomogeneous sample, we add them to the list of

TABLE 1

Summary of the Lensing Galaxy Parameters for the Best Systems

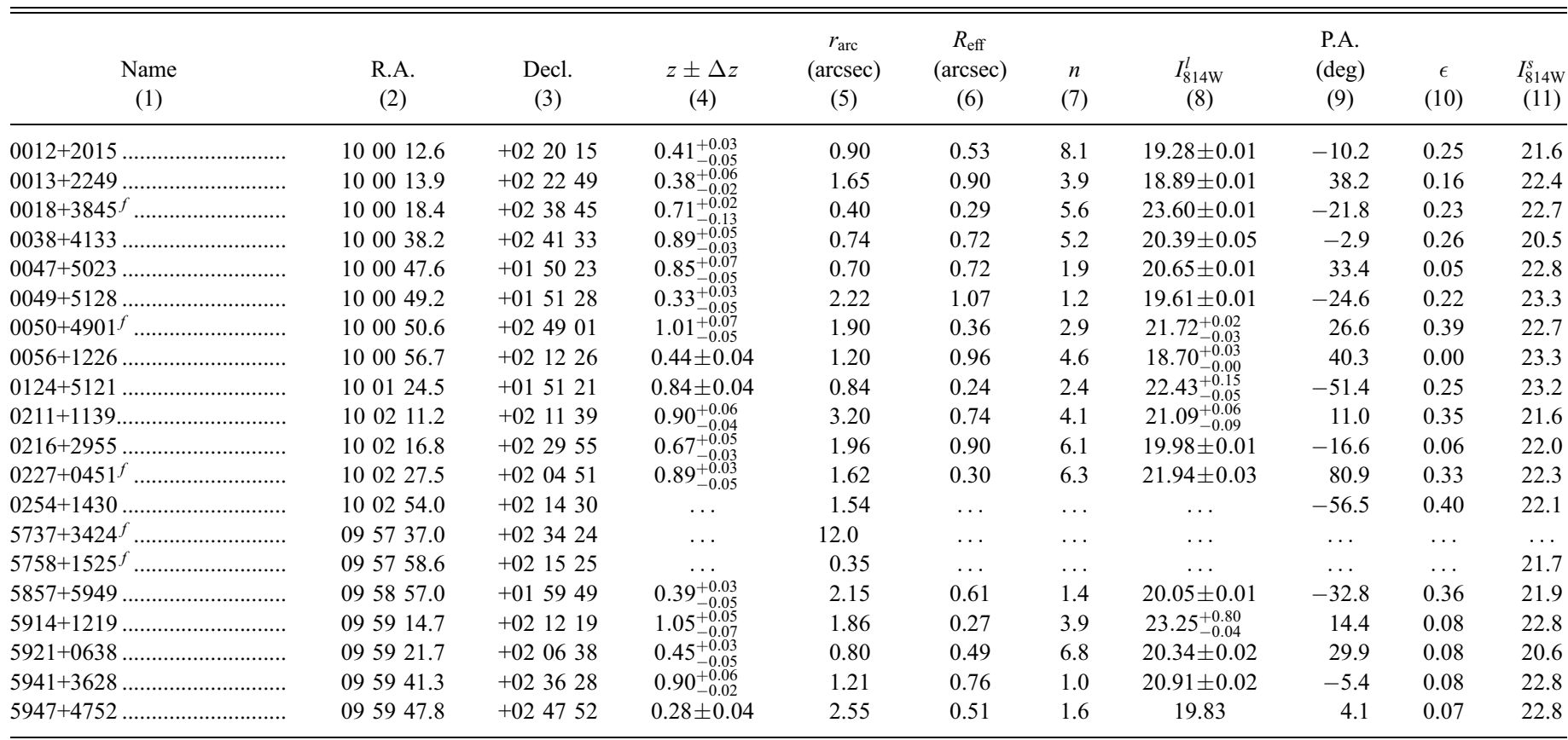

Notes.-Col. (1): Lens candidate name. A letter " $f$ " indicates that the object was found fortuitously. Cols. (2) and (3): Coordinates in J2000.0. Units of right ascension are hours, minutes, and seconds, and units of declination are degrees, arcminutes, and arcseconds. Col. (4): Photometric redshift of the lens and error bars (at $68 \%$ confidence level). Col. (5): Largest radius of the arc in arcseconds. Col. (6): Effective radius of the lensing galaxy from the surface brightness fitting, in arcseconds. Col. (7): Sersic profile index. Col. (8): Total magnitude of the lensing galaxy in the WFPC/F814W band. Col. (9): Lensing galaxy position angle. Col. (10): Galaxy ellipticity $\epsilon$. Col. (11): Magnitude of the brightest image in the WFPC/F814W band (in magnitudes per square arcsecond). 
TABLE 2

Summary of the Lensing Galaxy Parameters for the Single Arclet Systems

\begin{tabular}{|c|c|c|c|c|c|c|c|c|c|c|}
\hline $\begin{array}{c}\text { Cosmos Name } \\
\text { (1) }\end{array}$ & $\begin{array}{l}\text { R.A. } \\
(2)\end{array}$ & $\begin{array}{l}\text { Decl. } \\
\text { (3) }\end{array}$ & $\begin{array}{c}z \pm \Delta z \\
(4)\end{array}$ & $\begin{array}{c}r_{\operatorname{arc}} \\
(\operatorname{arcsec}) \\
(5)\end{array}$ & $\begin{array}{c}R_{\mathrm{eff}} \\
(\operatorname{arcsec}) \\
(6)\end{array}$ & $\begin{array}{c}n \\
(7)\end{array}$ & $\begin{array}{l}I_{814 \mathrm{~W}}^{l} \\
(8)\end{array}$ & $\begin{array}{l}\text { P.A. } \\
\text { (deg) } \\
(9)\end{array}$ & $\begin{array}{c}\epsilon \\
(10)\end{array}$ & $\begin{array}{l}I_{814 \mathrm{~W}}^{S} \\
(11)\end{array}$ \\
\hline $0009+2455 \ldots \ldots$ & 100009.7 & +022455 & $0.39 \pm 0.04$ & 2.63 & 0.70 & 3.7 & $19.49 \pm 0.01$ & -2.5 & 0.31 & 22.6 \\
\hline 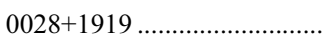 & 100028.6 & +021919 & $0.61_{-0.05}^{+0.03}$ & 1.87 & 0.45 & 1.7 & $20.34_{-0.03}^{+0.01}$ & 34.9 & 0.13 & 22.6 \\
\hline 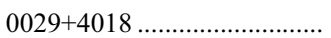 & 100029.6 & +024018 & $0.83_{-0.07}^{+0.05}$ & 2.99 & 0.83 & 2.6 & $18.94 \pm 0.02$ & 47.4 & 0.00 & 22.7 \\
\hline $0047+2931 \ldots \ldots \ldots \ldots \ldots \ldots \ldots \ldots \ldots$ & 100047.1 & +022931 & $0.32 \pm 0.04$ & 2.00 & 0.73 & 2.3 & $19.56 \pm 0.01$ & -38.4 & 0.29 & 21.6 \\
\hline 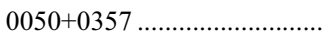 & 100050.8 & +020357 & $0.84 \pm 0.04$ & 0.66 & 0.31 & 2.8 & $22.31 \pm 0.02$ & -44.7 & 0.40 & 22.5 \\
\hline $0055+3821 \ldots \ldots \ldots$ & 100055.7 & +013821 & $0.66 \pm 0.06$ & 1.05 & 0.40 & 2.4 & $20.86 \pm 0.01$ & 10.3 & 0.14 & 21.8 \\
\hline $0056+2106 \ldots \ldots \ldots \ldots \ldots \ldots \ldots \ldots$ & 100056.7 & +022106 & $0.65_{-0.05}^{+0.03}$ & 1.75 & 0.34 & 3.4 & $21.21 \pm 0.04$ & 84.7 & 0.02 & 23.3 \\
\hline 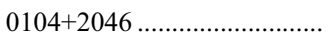 & 100104.9 & +022046 & $0.94 \pm 0.10$ & 3.54 & 0.11 & 3.8 & $24.27_{-0.05}^{+0.20}$ & -0.7 & 0.31 & 21.9 \\
\hline $0104+2501 \ldots \ldots \ldots \ldots \ldots \ldots \ldots \ldots \ldots$ & 100104.6 & +022501 & $0.22_{-0.02}^{+0.06}$ & 3.26 & $\ldots$ & $\ldots$ & $\ldots$ & $\ldots$ & $\ldots$ & 21.8 \\
\hline $0105+4531 \ldots \ldots \ldots \ldots \ldots \ldots \ldots \ldots \ldots$ & 100105.3 & +024531 & $0.44 \pm 0.04$ & 1.00 & 0.47 & 3.4 & $20.38 \pm 0.01$ & 15.9 & 0.00 & 22.3 \\
\hline 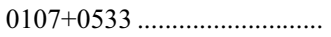 & 100107.9 & +020533 & $0.90 \pm 0.06$ & 1.71 & 0.24 & 3.2 & $21.83_{-0.07}^{+0.01}$ & -70.4 & 0.04 & 22.7 \\
\hline 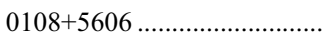 & 100108.0 & +015606 & $0.97_{-0.05}^{+0.07}$ & 1.25 & 0.20 & 1.8 & $22.81 \pm 0.02$ & -61.5 & 0.24 & 23.4 \\
\hline 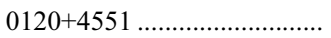 & 100120.2 & +014551 & $0.82 \pm 0.06$ & 1.28 & $\ldots$ & $\ldots$ & $\ldots$ & $\ldots$ & $\ldots$ & 22.9 \\
\hline $0148+2325 \ldots \ldots \ldots \ldots \ldots \ldots \ldots \ldots \ldots$ & 100148.1 & +022325 & $0.40 \pm 0.04$ & 1.43 & 1.27 & 2.9 & $18.81 \pm 0.01$ & -35.1 & 0.00 & 22.0 \\
\hline $0208+1422 \ldots \ldots$ & 100208.5 & +021422 & $0.41 \pm 0.04$ & 1.59 & 0.45 & 7.7 & $20.06_{-0.05}^{+0.07}$ & -52.0 & 0.00 & 22.0 \\
\hline 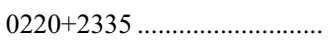 & 100220.2 & +022335 & $0.65_{-0.05}^{+0.03}$ & 1.04 & 0.60 & 1.7 & $20.99_{-0.08}^{+0.01}$ & 87.2 & 0.00 & 21.8 \\
\hline $0221+3440 \ldots \ldots \ldots \ldots \ldots \ldots \ldots \ldots$ & 100221.1 & +023440 & $0.41_{-0.05}^{+0.03}$ & 1.57 & 1.06 & 5.5 & $19.35 \pm 0.01$ & -22.7 & 0.04 & 22.3 \\
\hline $0236+4807 \ldots \ldots \ldots \ldots \ldots \ldots \ldots \ldots \ldots$ & 100236.0 & +024807 & $0.34 \pm 0.06$ & 1.27 & 0.68 & 1.8 & $20.15 \pm 0.01$ & 22.0 & 0.11 & 23.2 \\
\hline $0248+1422 \ldots \ldots \ldots \ldots \ldots \ldots \ldots \ldots \ldots \ldots \ldots \ldots \ldots$ & 100248.4 & +021422 & $0.60 \pm 0.04$ & 1.38 & 0.22 & 5.5 & $21.72_{-0.05}^{+0.11}$ & 18.8 & 0.24 & 22.8 \\
\hline $5748+5524 \ldots \ldots \ldots \ldots \ldots \ldots \ldots \ldots \ldots$ & 095748.0 & +015524 & $0.93_{-0.05}^{+0.07}$ & 2.21 & 0.33 & 1.4 & $22.15 \pm 0.02$ & 89.9 & 0.00 & 21.6 \\
\hline 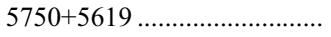 & 095750.7 & +015619 & $0.28 \pm 0.04$ & 0.73 & $\ldots$ & $\ldots$ & $\ldots$ & $\ldots$ & $\ldots$ & 21.8 \\
\hline $5752+2057 \ldots \ldots \ldots \ldots \ldots \ldots \ldots \ldots \ldots \ldots \ldots \ldots \ldots \ldots$ & 095752.1 & +022057 & $0.44 \pm 0.04$ & 0.98 & 0.51 & 1.0 & $20.27 \pm 0.01$ & 3.8 & 0.14 & 21.6 \\
\hline 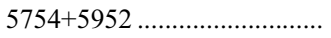 & 095754.1 & +015952 & $0.90_{-0.02}^{+0.06}$ & 1.33 & 0.22 & 6.9 & $22.23_{-0.05}^{+0.20}$ & -16.5 & 0.43 & 22.6 \\
\hline 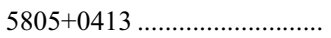 & 095805.6 & +020413 & $0.88 \pm 0.04$ & 1.61 & 0.12 & 4.8 & $23.00_{-0.02}^{+0.04}$ & -81.6 & 0.16 & 22.8 \\
\hline $5806+5809 \ldots \ldots$ & 095806.8 & +015809 & $0.32 \pm 0.04$ & 1.50 & 0.43 & 2.9 & $19.14_{-0.08}^{+0.02}$ & -13.6 & 0.00 & 21.7 \\
\hline $5821+4437^{f} \ldots$ & 095821.4 & +014437 & $0.67_{-0.03}^{+0.05}$ & 1.40 & 0.21 & 3.5 & $21.84_{-0.11}^{+0.08}$ & 00.0 & 0.28 & 22.9 \\
\hline $5829+3734^{f} \ldots \ldots$ & 095829.9 & +013734 & $0.51_{-0.03}^{+0.05}$ & 2.97 & 0.46 & 1.6 & $19.95_{-0.03}^{+0.00}$ & -0.1 & 1.15 & 22.3 \\
\hline $5831+4332^{f} \ldots \ldots \ldots \ldots \ldots \ldots \ldots \ldots$ & 095831.0 & +014332 & $\ldots$ & 1.17 & $\ldots$ & $\ldots$ & $\ldots$ & $\ldots$ & $\ldots$ & 21.0 \\
\hline $5841+4646 \ldots \ldots \ldots \ldots \ldots \ldots \ldots \ldots \ldots$ & 095841.4 & +024646 & $0.36 \pm 0.04$ & 0.93 & 0.47 & 0.9 & $19.65 \pm 0.01$ & 21.1 & 0.12 & 21.0 \\
\hline 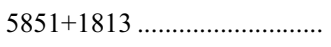 & 095851.9 & +021813 & $0.29_{-0.05}^{+0.03}$ & 2.57 & $\ldots$ & $\cdots$ & $\ldots$ & $\cdots$ & $\cdots$ & 21.9 \\
\hline $5856+4755 \ldots \ldots \ldots \ldots \ldots \ldots \ldots \ldots \ldots \ldots \ldots \ldots \ldots \ldots$ & 095856.1 & +024755 & $0.32 \pm 0.04$ & 2.97 & 0.17 & 1.1 & $19.54 \pm 0.01$ & 48.8 & 0.39 & 22.9 \\
\hline 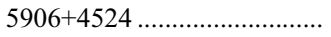 & 095906.5 & +024524 & $0.37_{-0.05}^{+0.03}$ & 1.50 & $\ldots$ & $\ldots$ & $\ldots$ & $\ldots$ & $\ldots$ & 21.6 \\
\hline $5919+3853 \ldots \ldots+\cdots \cdots \cdots \cdots \cdots$ & 095919.4 & +013853 & $0.40 \pm 0.04$ & 2.35 & 0.26 & 1.9 & $20.59_{-0.02}^{+0.01}$ & -15.7 & 0.08 & 22.7 \\
\hline $5924+0852 \ldots \ldots \ldots \ldots \ldots \ldots \ldots \ldots$ & 095924.7 & +020852 & $0.93_{-0.05}^{+0.07}$ & 0.97 & 0.17 & 1.6 & $23.09_{-0.02}^{+0.27}$ & -9.8 & 0.01 & 22.7 \\
\hline 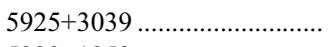 & 095925.8 & +023039 & $0.91_{-0.05}^{+0.07}$ & 0.98 & 0.15 & 1.2 & $23.16_{-0.04}^{+0.05}$ & 89.9 & 0.38 & 23.2 \\
\hline $5929+1352 \ldots \ldots \ldots \ldots \ldots \ldots \ldots \ldots \ldots$ & 095929.9 & +021352 & $0.36 \pm 0.04$ & 2.46 & $\ldots$ & $\ldots$ & & $\ldots$ & $\ldots$ & 22.0 \\
\hline $5929+4553 \ldots \ldots \ldots \ldots \ldots \ldots \ldots \ldots$ & 095929.0 & +014553 & $0.54_{-0.02}^{+0.06}$ & 1.56 & 0.67 & 2.1 & $19.99 \pm 0.01$ & -23.2 & 0.06 & 22.3 \\
\hline 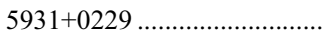 & 095931.1 & +020229 & $0.32 \pm 0.04$ & 1.48 & 0.81 & 1.9 & $19.20_{-0.02}^{+0.00}$ & 89.9 & 0.01 & 21.6 \\
\hline $5932+1018 \ldots \ldots \ldots \ldots \ldots \ldots \ldots \ldots$ & 095932.0 & +021018 & $0.92_{-0.06}^{+0.04}$ & 0.68 & 0.40 & 1.2 & $21.92_{-0.03}^{+0.12}$ & 19.9 & 0.22 & 22.6 \\
\hline $5936+3621 \ldots \ldots \ldots \ldots \ldots \ldots \ldots \ldots$ & 095936.7 & +023621 & $0.25_{-0.05}^{+0.03}$ & 2.40 & 0.71 & 0.9 & $19.14_{-0.01}^{+0.02}$ & -42.4 & 0.15 & 22.1 \\
\hline 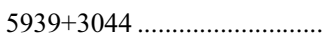 & 095939.1 & +023044 & $0.73_{-0.05}^{+0.03}$ & 0.70 & 0.58 & 1.7 & $20.08_{-0.05}^{+0.01}$ & 76.9 & 0.00 & 22.9 \\
\hline $5940+3253 \ldots \ldots \ldots \ldots \ldots \ldots \ldots \ldots \ldots$ & 095940.4 & +023253 & $0.41_{-0.05}^{+0.03}$ & 1.53 & 0.51 & 1.4 & $19.45 \pm 0.01$ & 58.4 & 0.40 & 22.5 \\
\hline 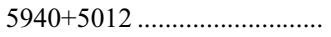 & 095940.0 & +025012 & $0.36 \pm 0.04$ & 1.97 & $\ldots$ & $\ldots$ & $\ldots$ & $\ldots$ & $\ldots$ & 21.8 \\
\hline $5942+2829 \ldots \ldots$. & 095942.7 & +022829 & $0.72 \pm 0.04$ & 1.39 & 0.31 & 1.6 & $21.88_{-0.05}^{+0.03}$ & 34.0 & 0.04 & 22.8 \\
\hline $5943+2816 \ldots \ldots \ldots \ldots \ldots \ldots \ldots \ldots$ & 095943.1 & +022816 & $0.39_{-0.03}^{+0.05}$ & 1.64 & 0.69 & 1.3 & $19.43 \pm 0.01$ & 79.9 & 0.01 & 22.4 \\
\hline 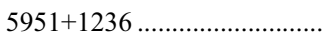 & 095951.0 & +021236 & $0.46_{-0.02}^{+0.06}$ & 1.53 & 1.21 & 2.2 & $18.66 \pm 0.01$ & 20.9 & 0.29 & 21.9 \\
\hline 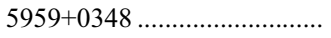 & 095959.7 & +020348 & $0.82 \pm 0.06$ & 1.20 & 0.20 & 3.5 & $21.62 \pm 0.04$ & -49.2 & 0.20 & 22.8 \\
\hline
\end{tabular}

Notes.-Col. (1): Lens candidate name. Cols. (2) and (3): Coordinates in J2000.0. Units of right ascension are hours, minutes, and seconds, and units of declination are

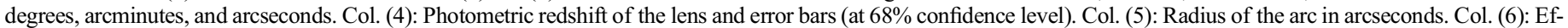

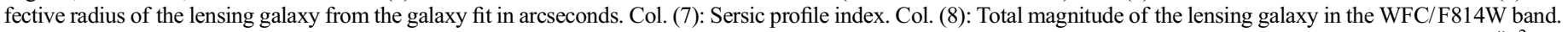

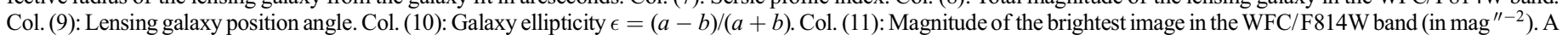
letter " $f$ " indicates that the object was found fortuitously.

strong lensing systems for completeness. Those systems are identified in Tables 1 and 2 .

\subsection{Visual Inspection of the ACS Images}

We visually inspect a postage-stamp $10^{\prime \prime} \times 10^{\prime \prime}$ ACS image surrounding all galaxies in the $\mathrm{P}$ catalog, looking for lensed features (tangential arcs or multiple images). The search box size limits us to $r_{\text {arc }} \lesssim 5^{\prime \prime}$. Consequently, this puts an upper limit on the mass scale for the lens, which excludes clusters as a possible main deflector. This inspection was performed by five of the coauthors using the DS9 FITS image visualization tool.

Our strategy reduced the 9452 galaxies in the $\mathrm{P}$ catalog to an $\mathrm{E}$ catalog of 337 candidates, with intentionally loose selection criteria to avoid excluding any real lenses at this stage.

\subsection{Pseudocolor Images}

A color image is always useful to verify potential lens candidates. Indeed, gravitational lensing is achromatic; thus, a gravitational 

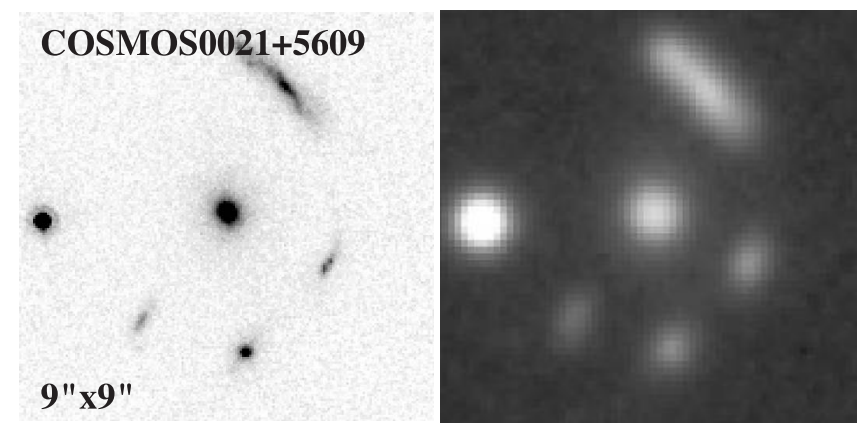

COSMOS0157+3517

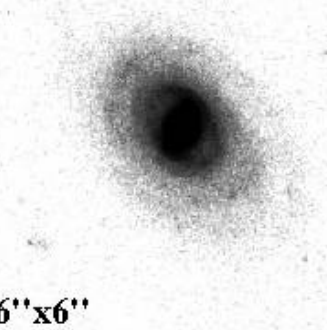

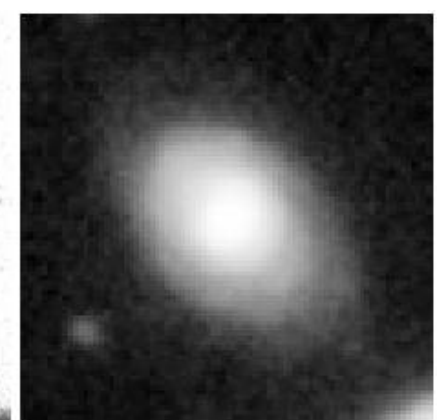

FIG. 2.-Examples of strong lens candidates ruled out by the pseudocolor image test. North is at the top of the figure, and east is to the left. The left stamp images are the ACS I-band images of the systems. The right stamp images are the color images. [See the electronic edition of the Supplement for a color version of this figure.]

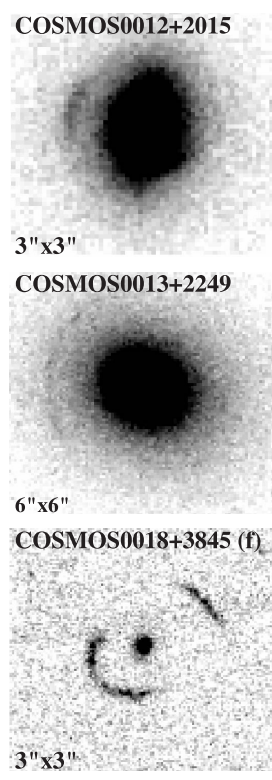

COSMOS0038+4133

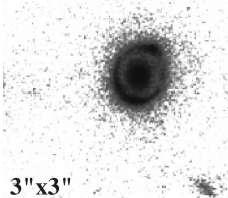

COSMOS0047+5023

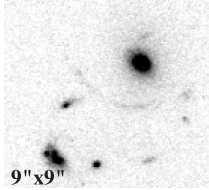

COSMOS0049+5128

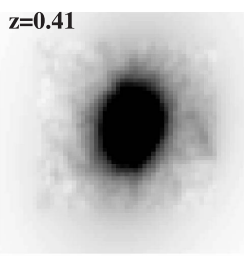

$\mathrm{z}=\mathbf{0 . 3 8}$

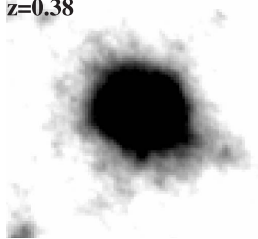

$\mathrm{z}=\mathbf{0 . 7 1}$

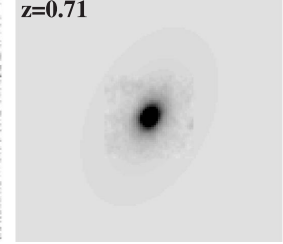

$\mathrm{z}=\mathbf{0 . 8 9}$

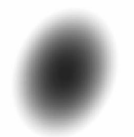

$\mathrm{z}=\mathbf{0 . 8 5}$

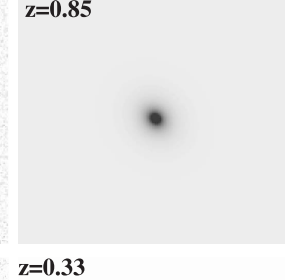

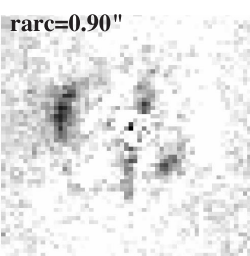
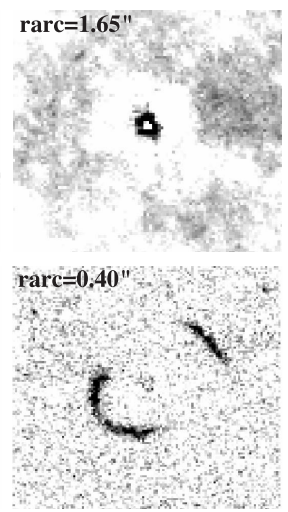

rarc $=0.74$

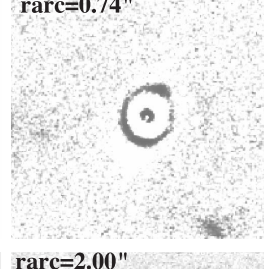

$\operatorname{rarc}=\mathbf{2 . 0 0 "}$

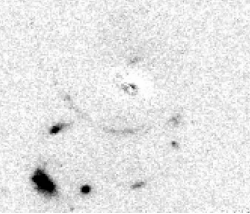

$\operatorname{rarc}=2.22$
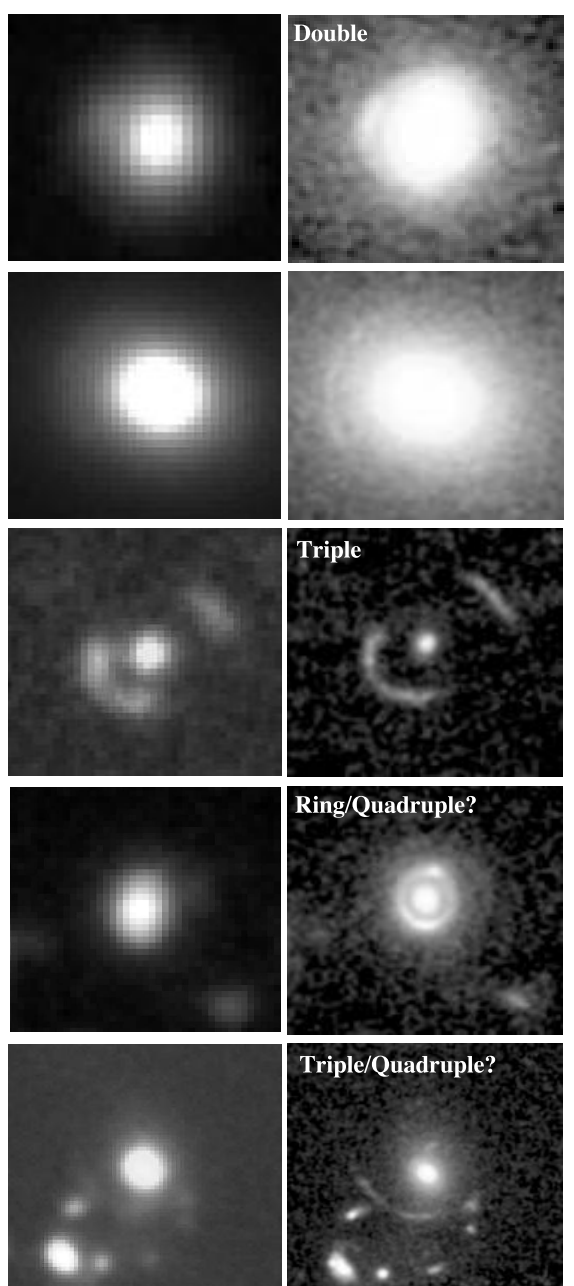

Triple/Quadruple?
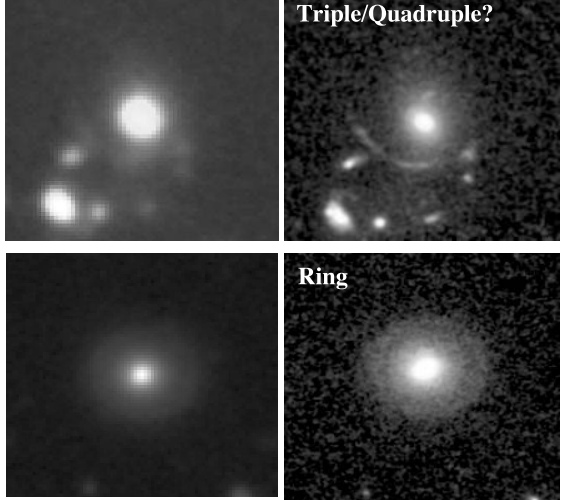

Ring

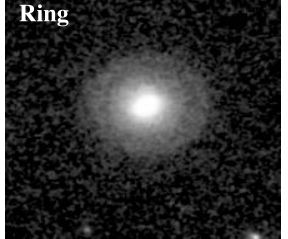

FIG. 3.-First strong lensing candidates in the COSMOS field: the best systems. From left to right: The first panel is the ACS I-band image of the lens. The letter "f "

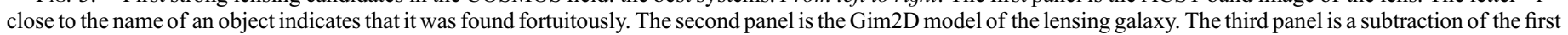

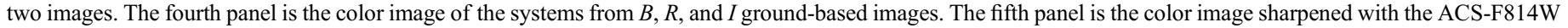

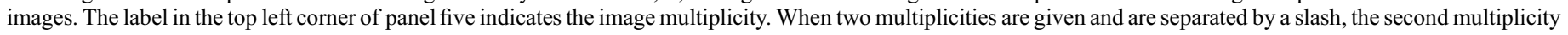

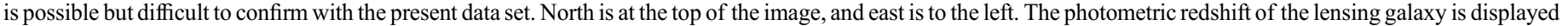

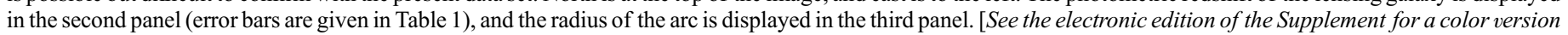
of this figure.] 


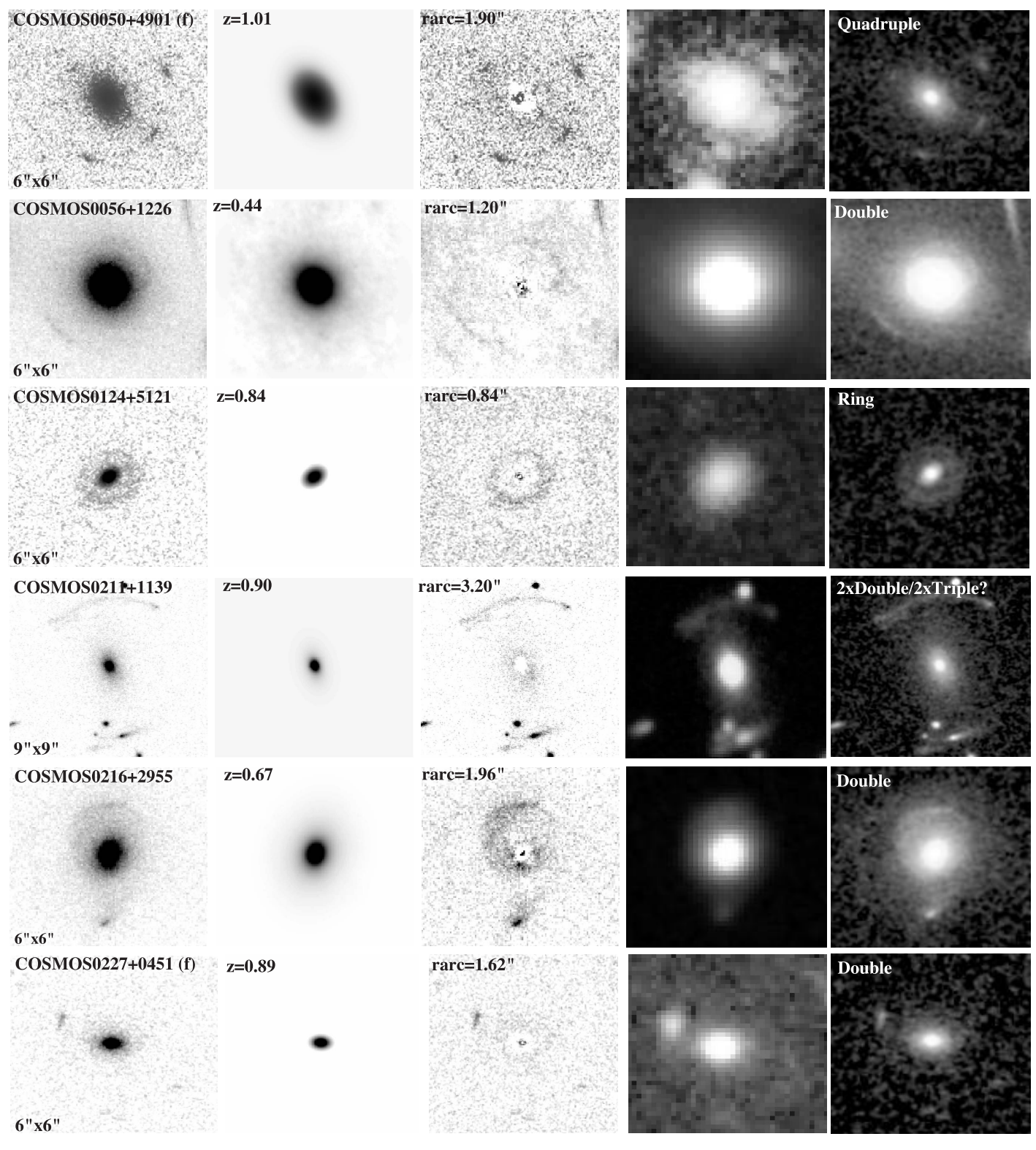

FIG. 3-Continued

arc or multiple images systems should have a consistent color across the image(s). Furthermore, the color of a lensed galaxy will only rarely have the same color as the main lensing galaxy. This color test hence provides a good way to discriminate false strong lensing cases. However, with our particular combination of ground- and space-based data (especially when the seeing approaches $1^{\prime \prime}$ ), the color test is only useful for arcs more distant than $\sim 0.5^{\prime \prime}$ from the lensing galaxy because of strong contamination from the wings of the lensing galaxy. For the 337 systems in the E catalog, we produced pseudocolor images made from the "best PSF" stacks of the $B, r$, and $i$ Subaru images, using the RGB feature in the DS9 software, as well as ground-based images sharpened with the ACS images. The color images use the ACS F814W data as an illumination map and the Subaru $B_{J}, r^{+}$, and $z^{+}$images as a color map. To achieve this each Subaru image is divided by the average of the three Subaru images and then multiplied by the ACS F814W image. This preserves the flux ratio between images while replacing the overall illumination pattern with the F814W data. Each image is then smoothed by a 1 pixel Gaussian to reduce the noise and divided by $\lambda^{2}$ to enhance the color difference between star-forming and passive galaxies. The processed $B_{J}, r^{+}$, and $z^{+}$images are then assigned to the blue, green, and red channels, respectively. The resulting images have the high spatial resolution of the ACS imaging but color gradients at ground-based resolution. Of the 337 candidates, 67 have plausible colors for strong lensing systems. The color images are displayed in Figures 3-5. For illustrative purposes, two objects that were rejected at this stage are shown in Figure 2. The system displayed in the top panel of Figure 2 shows two arclike features in the ACS image: a giant arc located northwest of the main galaxy and its candidate counterpart located to the southwest. The pseudocolor image reveals that the "arcs" have very different colors, indicating that they are not images of the same source; neither are likely to be distorted images of background galaxies. Pronounced, arclike features are also observed in the ACS image shown in the bottom panel of Figure 2, northeast of the main galaxy bulge. With the single-color ACS data set only, it would not be clear whether these features were part of the galaxy's spiral arms or distorted images of a background object. The pseudocolor image shows blue knots of star formation all around 


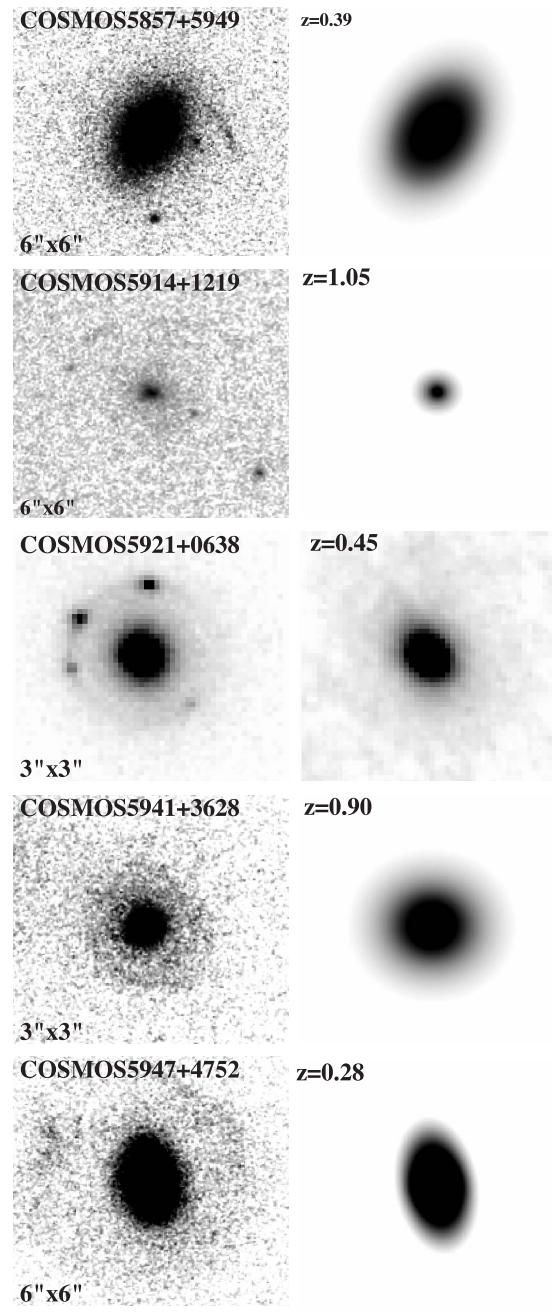

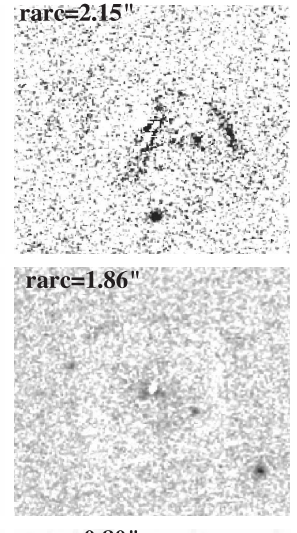
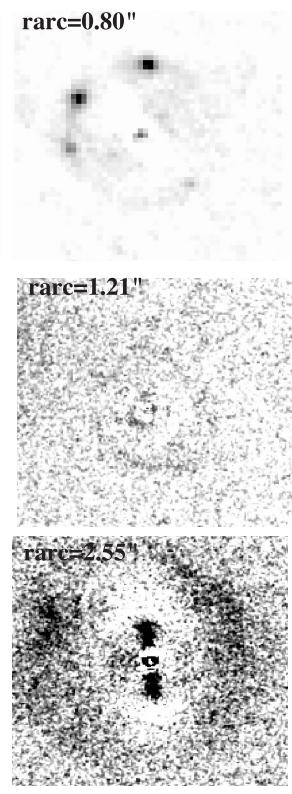

FIG. 3-Continued
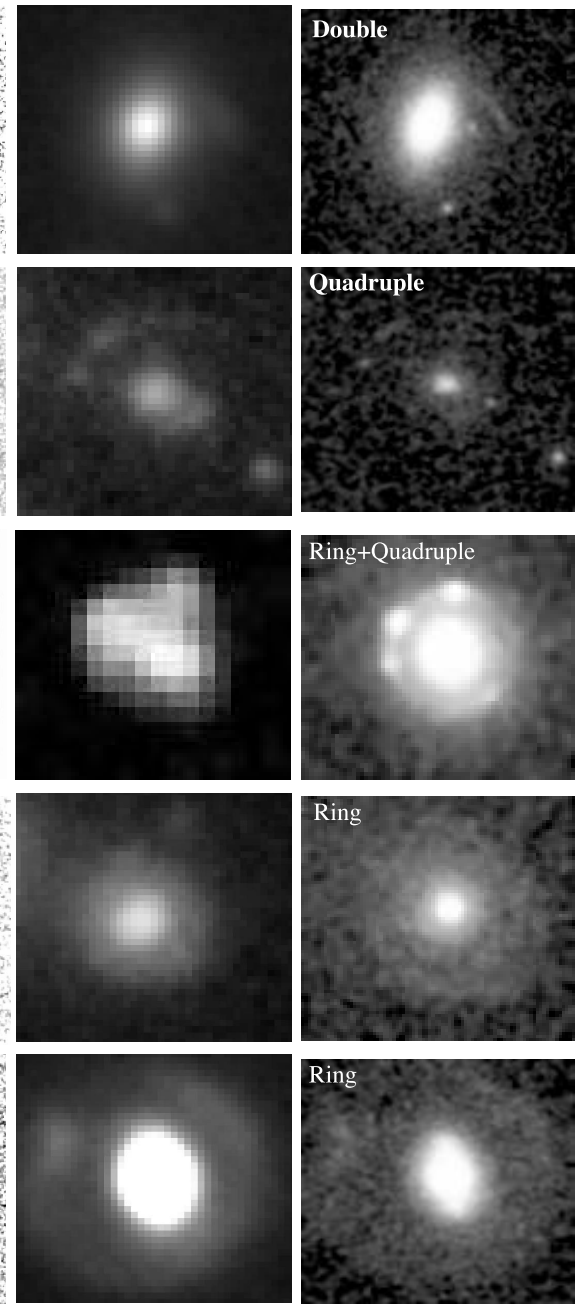

the disk of this galaxy, suggesting that the arclike features are most probably genuine regions of the main galaxy.

\subsection{The Lensing Galaxy Fit and Subtraction}

Fitting and extracting the foreground lens galaxy light from the images reveals the shape of the more distant, lensed object. For this work, a robust two-dimensional fit to the galaxy surface brightness distribution is derived using GIM2D (Simard 1998; Marleau \& Simard 1998). We adopt a Sèrsic bulge plus exponential disk parameterization to describe the two-dimensional surface brightness distribution of the lensing galaxy light profile. The Sérsic law is parameterized by means of the total flux in the bulge, the Sérsic index $n$, the bulge ellipticity $(\epsilon=1-b / a)$, the position angle of the bulge, and the effective radius of the bulge $R_{\text {eff }}$. The exponential profile depends on the photometric disk total flux, the disk scale length, the disk position angle, and the disk inclination. The GIM2D software finds the best-fitting values for all of these parameters and provides them as an output of the decomposition process.

In addition to the structural parameters for each object, we calculate a model image of each galaxy, which is convolved with a point-spread function before comparison with real data. An image of the residuals is obtained by the subtraction of the model from the science image. We present the result of the galaxy fits in Tables 1 and 2 and in Figures 3 and 4. In most cases, the arc candidates appear clearly after the galaxy model is subtracted (panel 3 in those figures).

In 10 cases, the modeling and subtraction of the lens galaxy proves difficult. The problems encountered include irregular galaxy morphology and compact galaxy size. We display the ACS and color images of these systems in Figure 5. In addition, the system COSMOS $5737+3424$ was discovered serendipitously in a part of the ground-based data set not covered by the ACS images. We present just its pseudocolor image in Figure 5.

\section{RESULTS OF THE STRONG LENS SEARCH}

The location of lens candidates in the absolute $V$-band magnitude versus redshift plane are indicated by plus signs in Figure 1.

We divide the sample in two subsamples: the multiple arcs and long curved image systems, which we refer to hereafter as the "best systems," and less certain single arclet systems. Given their distinctive shape, the multiple images and long curved arcs have a greater probability of being genuine strong lenses relative to the single arclet systems. Throughout the rest of this paper we therefore restrict our study to the sample of best systems.

The stamp images of the best systems are shown in Figures $3 a-$ $3 c$ and 5 and discussed in $\S 4$.1. Images of the single arclet systems are displayed in Figures $4 a-4 g$ and 5 and discussed in $\S$ 4.2. Their characteristics are given in Tables 1 and 2. In Figure 6 we have reported the distribution of the arc radius of the 


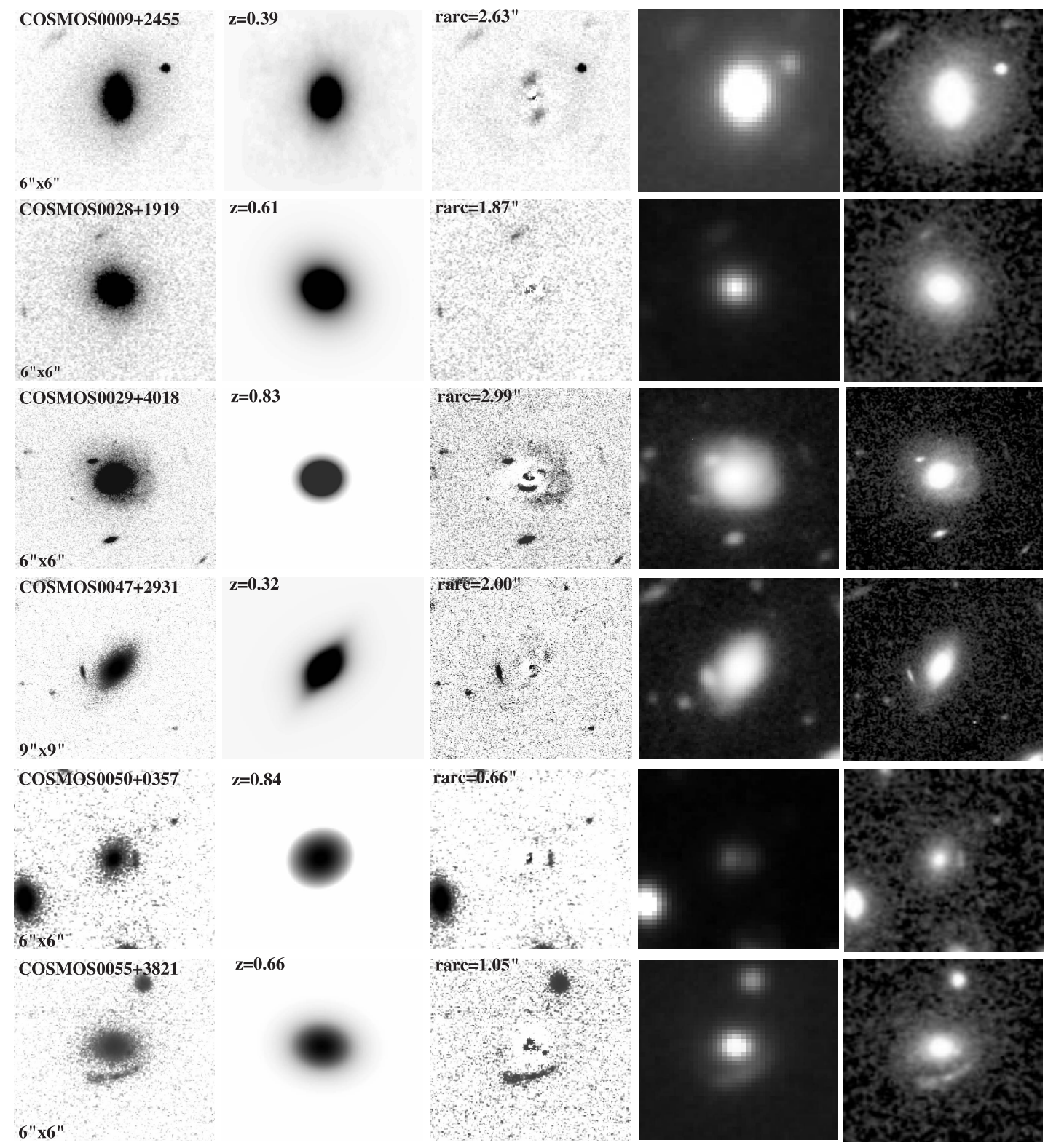

Fig. 4. - First strong lensing candidates in the COSMOS field: the single arclet systems. From left to right: The first panel is the ACS I-band image of the lens. The letter " $\mathrm{f}$ " close to the name of an object indicates that it was found fortuitously. The second panel is the Gim2D model of the lensing galaxy. The third panel is the difference between the two first images. The fourth panel is the color image of the systems from $B, R$, and $I$ ground-based images. The fifth panel is the color image sharpened with the ACS-F814W images. North is at the top of the image, and east is to the left. The photometric redshift of the lensing galaxy is displayed in the second panel (error bars are given in Table 2), and the radius of the arc is displayed in the third panel. [See the electronic edition of the Supplement for a color version of this figure.] 


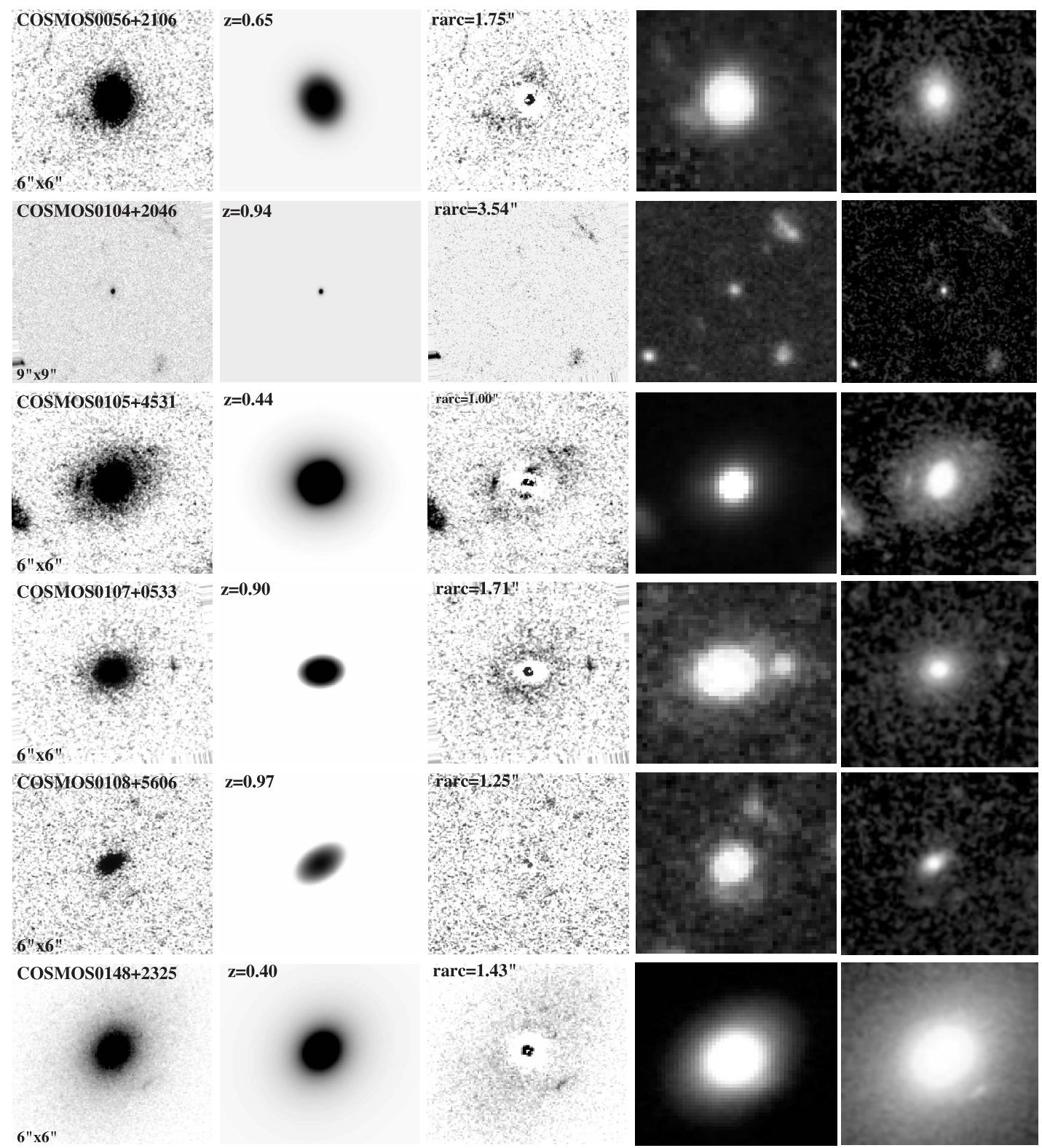

FIG. 4-Continued 

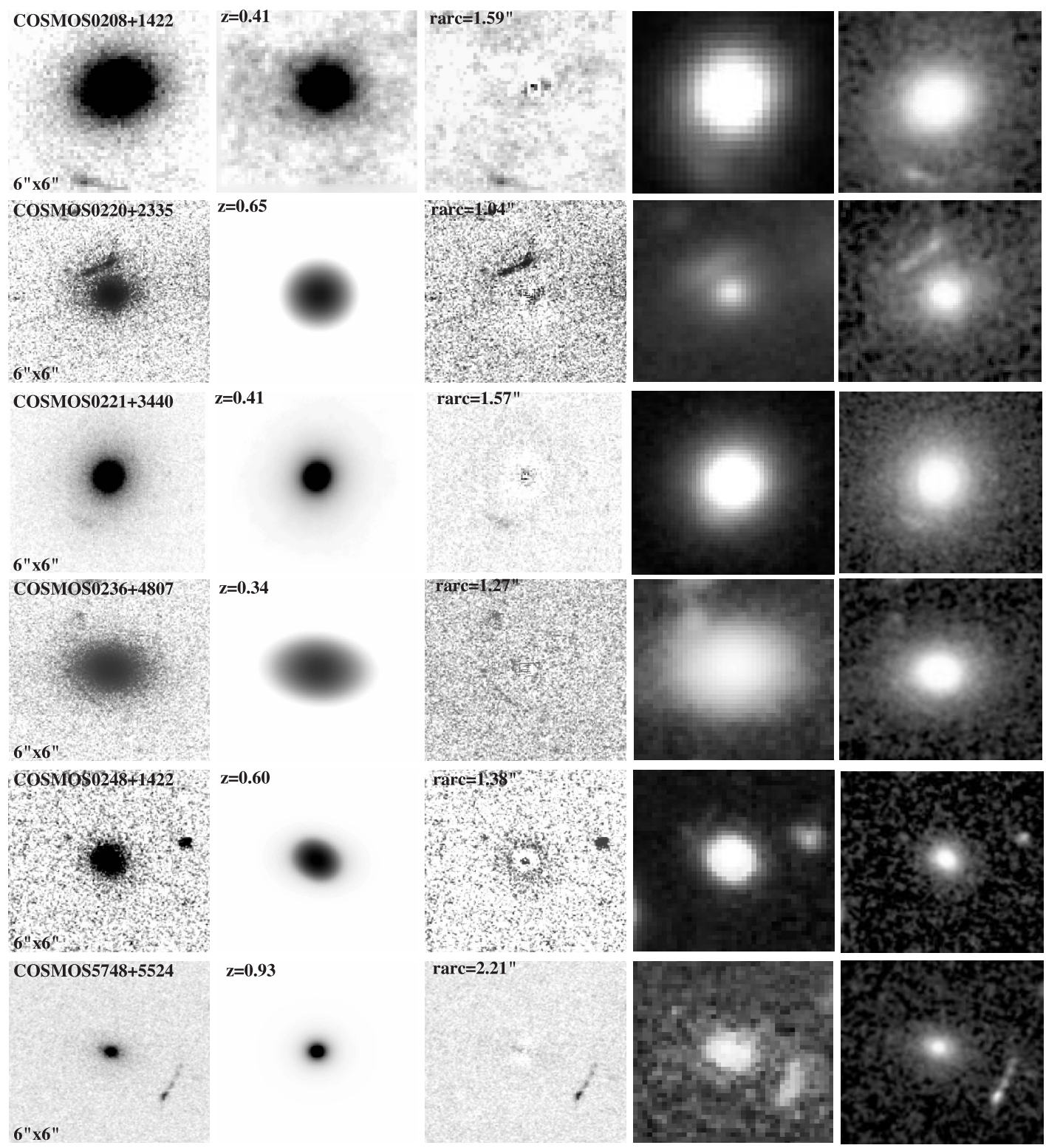

Fig. 4-Continued 


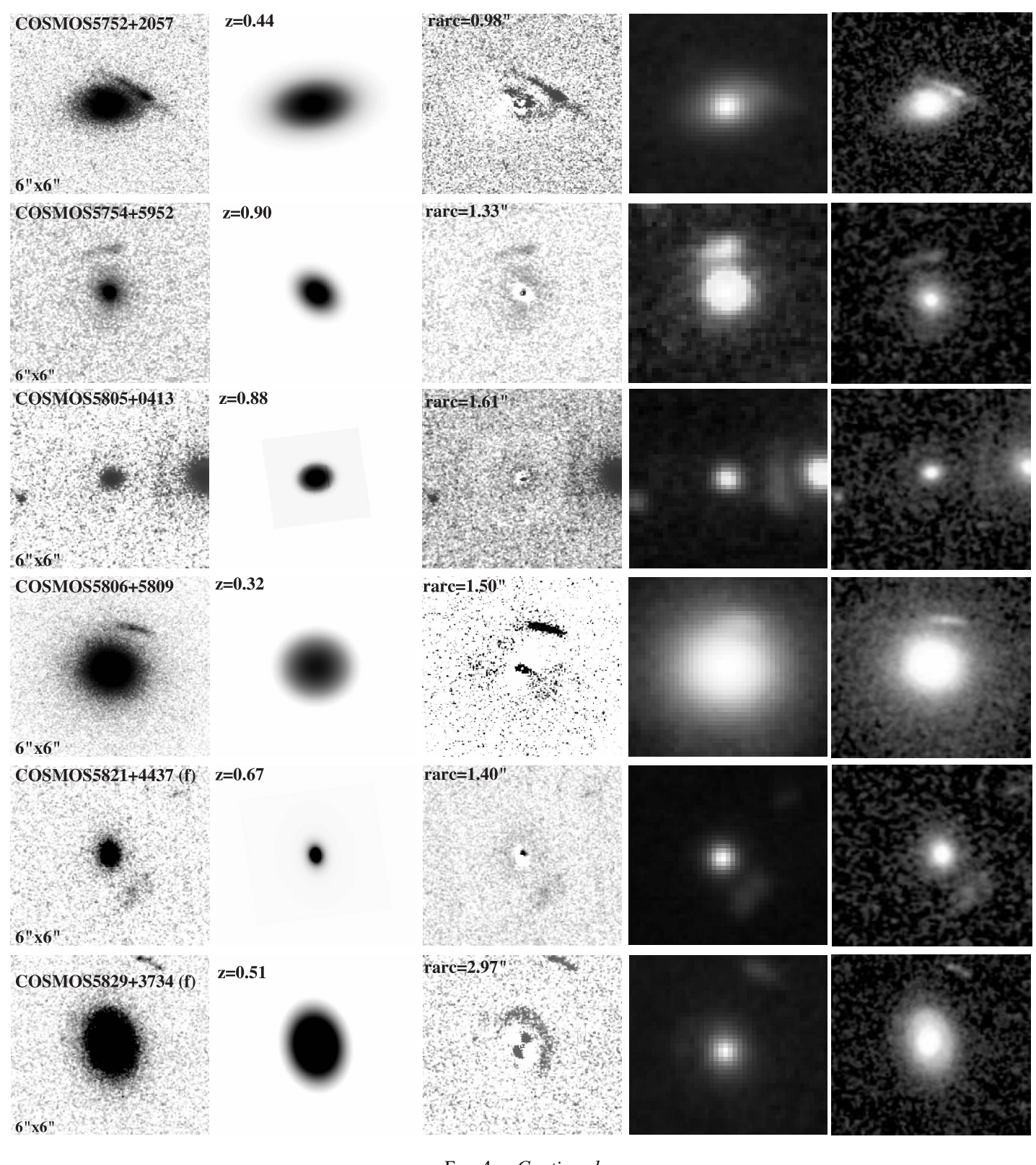

FIg. 4-Continued 


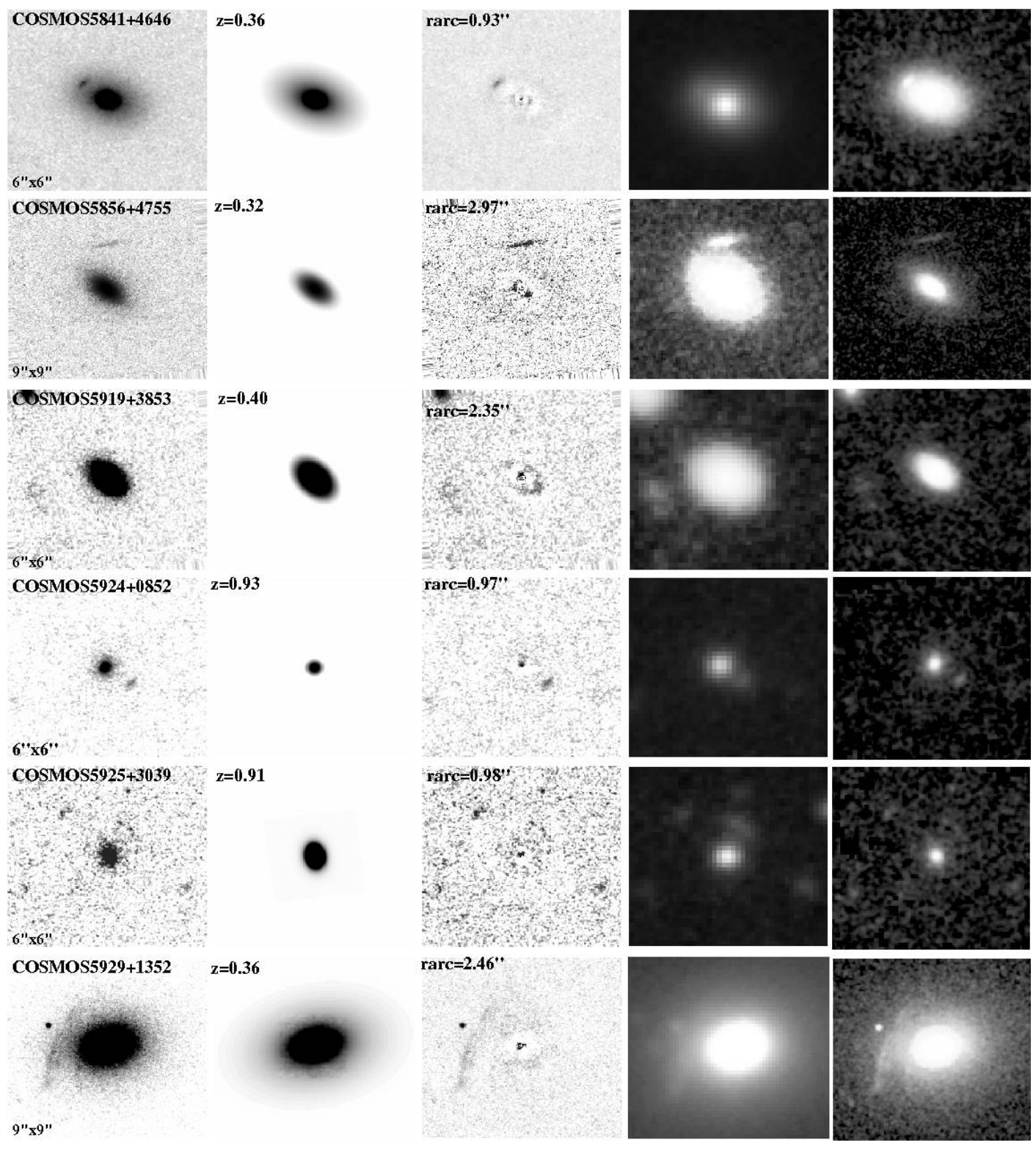

FIG. 4-Continued 


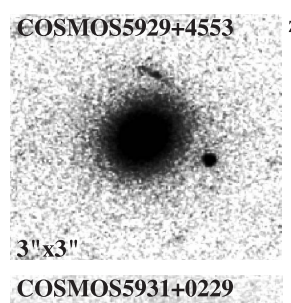

$\mathrm{z}=\mathbf{0 . 5 4}$

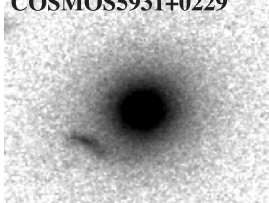

$\mathrm{z}=\mathbf{0 . 3 2}$

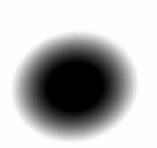

3 "x3"

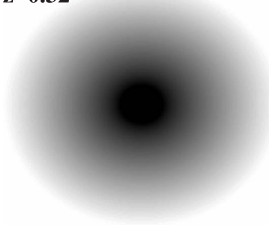

COSMOS5932+1018

$\mathrm{z}=0.92$

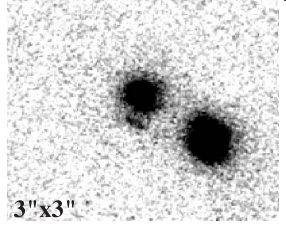

COSMOS5936+3621

$\mathrm{z}=\mathbf{0 . 2 5}$
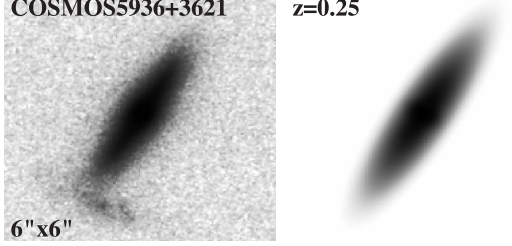

COSMOS5939+3044

$\mathrm{z}=\mathbf{0 . 7 3}$
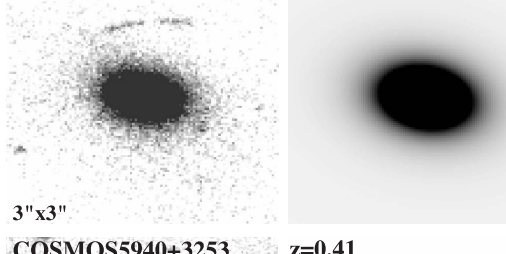

COSMOS5940+3253

- $z=0.41$
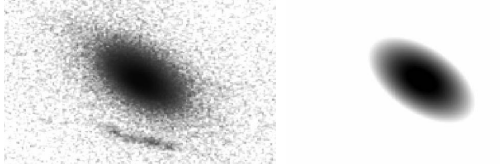

6"x6"
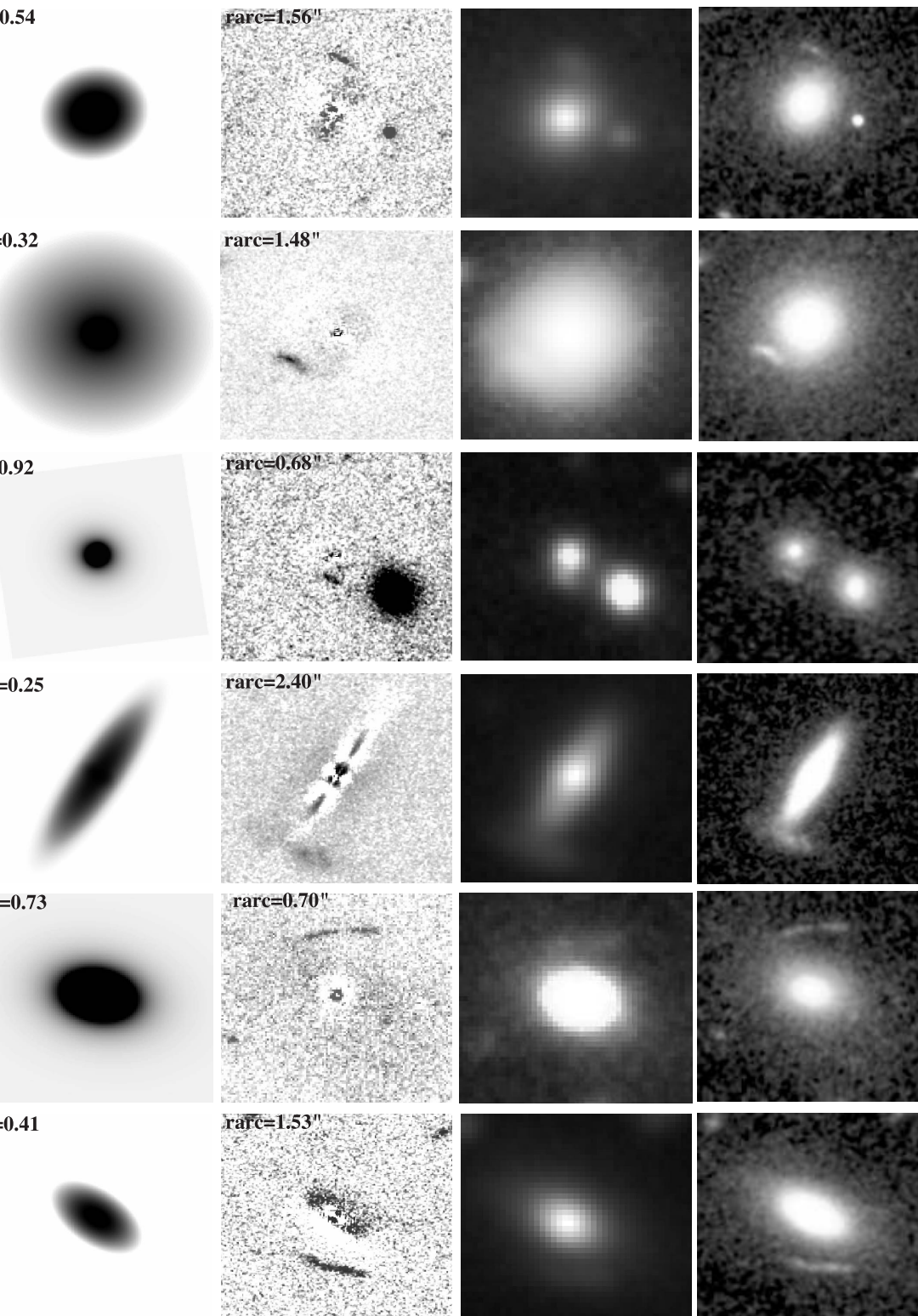

$\operatorname{rarc}=1.48$
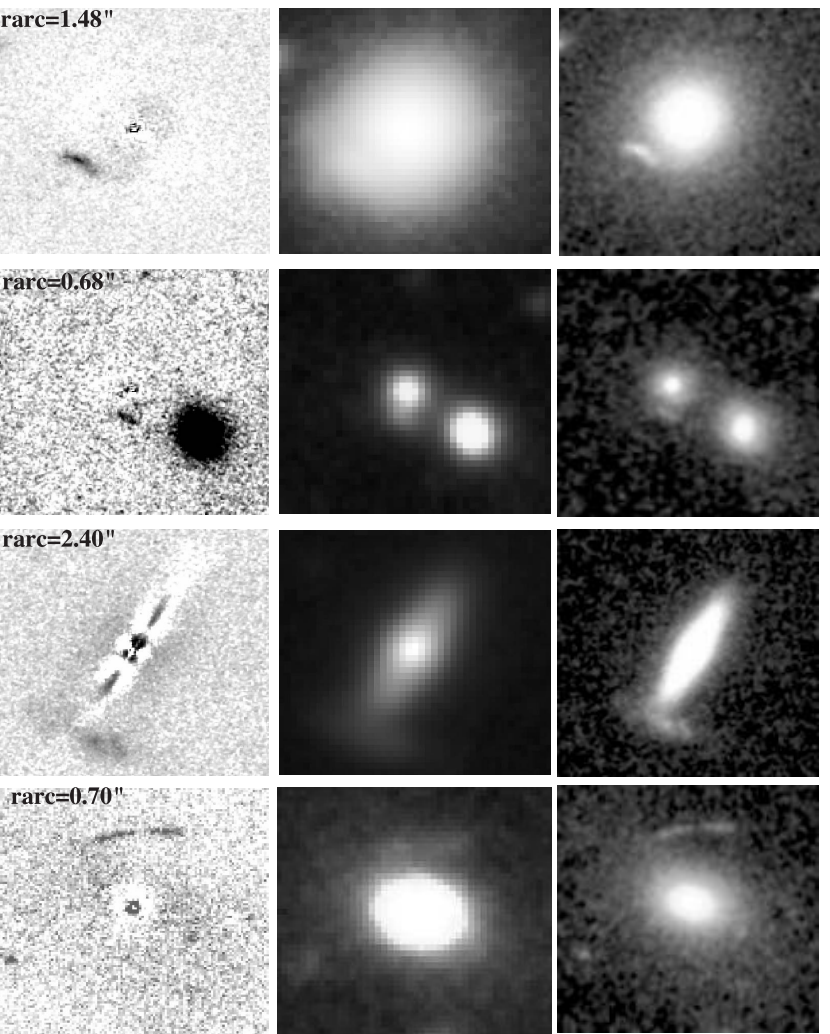

FIG. 4-Continued
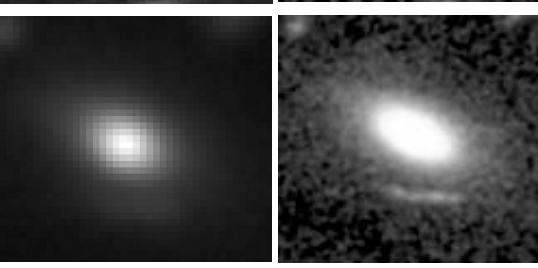


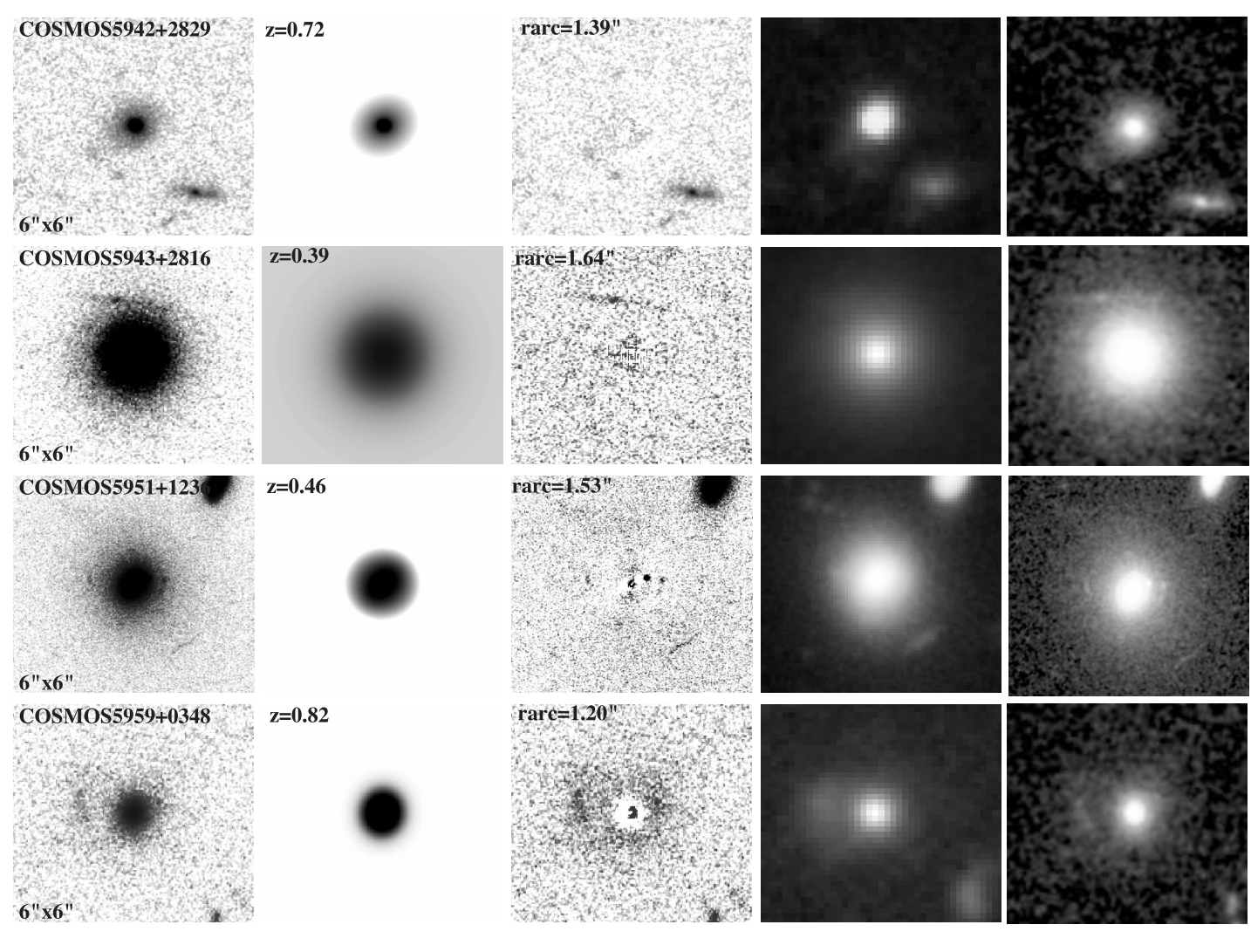

FIG. 4-Continued

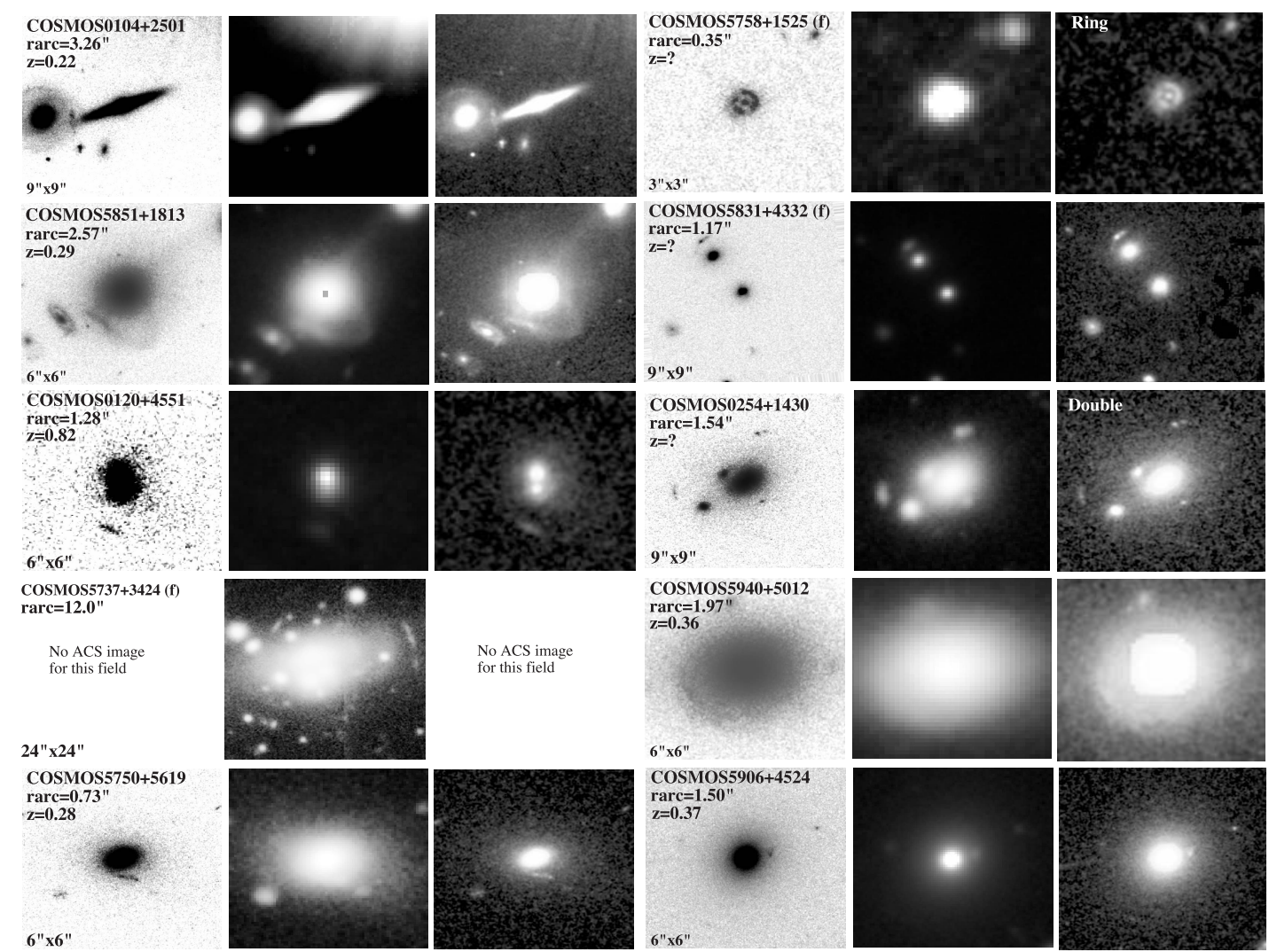

FIG. 5.-Other strong lensing candidates. For these systems, no satisfying fit of the lensing galaxy was possible. Therefore, we display only the ACS I-band image of

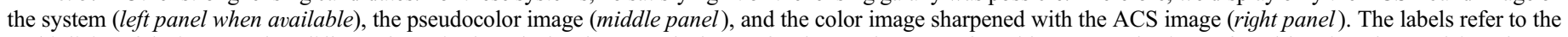

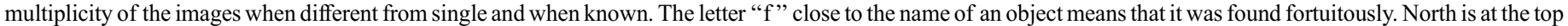
of the image, and east is to the left. [See the electronic edition of the Supplement for a color version of this figure.] 


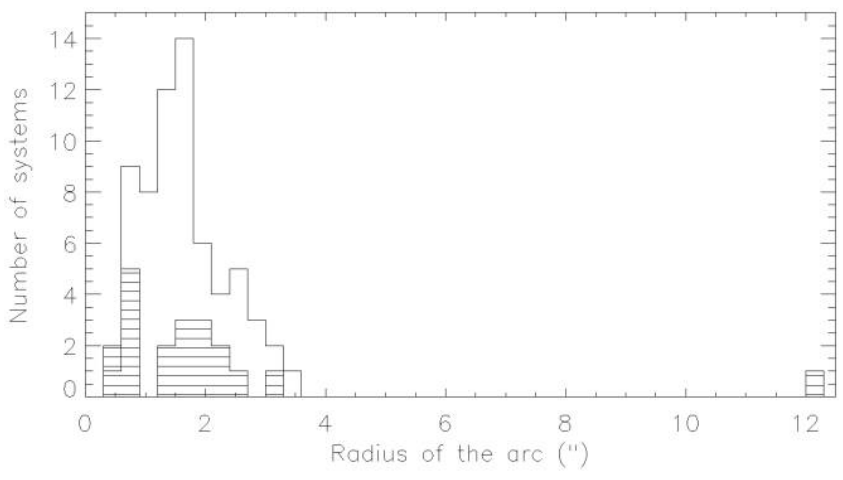

FIG. 6.-Distribution of the arc radii in the sample of COSMOS strong lens candidates (solid histogram). The striped histogram indicates the distribution of the best systems.

COSMOS strong lens candidates. Most systems have an arc radius $\lesssim 2^{\prime \prime}$.

\subsection{The Best Systems}

In 20 cases we have detected systems with a single long curved image or with two or more arcs of similar color symmetrically located around the lensing galaxy or with ringlike features. Their coordinates and characteristics are listed in Table 1. We comment on the most interesting or/and less trivial systems hereafter.

A bright arclike feature is clearly visible in the ACS image of the system COSMOS 0012+2015. The counterarc becomes visible after subtraction of the lensing galaxy profile, southwest of the center of the lens galaxy.

The system COSMOS $0018+3845$ is most probably a triply or possibly quadruply imaged background galaxy. The northwest arc would be a single image of the galaxy, while the southeast arc would be a composite of two or three images of the same galaxy.

The system COSMOS $0038+4133$ is a complete Einstein ring candidate, formed by the distorted image of a single background galaxy.

Four systems show ringlike structures around a central elliptical object (COSMOS 0049+5128, COSMOS 0124+5121, COSMOS $5941+3628$, and COSMOS 5947+4752). In those cases, it is difficult to conclude without any spectroscopic data whether the ring is due to gravitational lensing or if it is a genuine part of the central galaxy (like a ring-galaxy or a shell). The main argument in favor of them being lens candidates is the strong color difference between the elliptical central objects and the ring features, suggesting that they are not physically related.

There are probably four images of a background source in the candidate system COSMOS $0050+4901$. Three images are combined in an arc southwest of the lensing galaxy, while a counterpart image is visible northeast of the galaxy.

The system COSMOS 0211+1139 displays two giant arcs, each with different colors in the Subaru image. They are probably distorted images of two different background galaxies.

The pseudocolor image of COSMOS0216+2955 shows a strong color difference between the galaxy and the potential arcs. Nevertheless, the galaxy subtraction shows features that are not fitted by the Sersic profile. They seem to be linked to the galaxy core. Hence, there are two possible explanations for this system: either it is actually a strong lens, and the lensing galaxy is a spiral galaxy, or the two arcs observed are part of the arms of a spiral galaxy.

The system COSMOS0254+1430 has two lensing galaxies at very short distance. We have not been able to make a clean galaxy light profile fit. The two arclets located northeast and southwest are clearly visible in the color image.

In the system COSMOS 5737+3424, we observe at least three giant arclike features around the central galaxies of an identified XMM-Newton detected cluster (Finoguenov et al. 2007).

COSMOS $5921+0638$ is a quadruply imaged lensed quasar or very compact source. An Einstein ring is clearly visible in the $\mathrm{ACS} / \mathrm{F} 814 \mathrm{~W}$ band image.

\subsection{The Single Arclet Systems}

Forty-seven of the discovered lens candidates have a single arclet identified in both the ACS and the color images. In these cases, no counterimage for these arcs could be found in the residuals of the galaxy-subtracted image. As observed on the Subaru data, the arclets are generally much bluer than the lensing galaxies and are likely to be only weakly distorted by the galaxy. Their coordinates and characteristics are give in Table 2 . We comment below on a few interesting systems.

The system COSMOS $0009+2455$ shows two elongated features symmetrically distributed around the elliptical galaxy. The two features have slightly different colors; therefore, if the system is a genuine lens, the sources are probably two different objects.

The arclet in the system COSMOS $0028+1919$ is located west of the lensing galaxy and appears in blue in the color image.

In the system COSMOS 5805+0413, a giant blue arclet appears between two galaxies. The arc is slightly curved in direction of the western galaxy, but there are no counterpart images around any of the two galaxies. The parameters of the early spectral type galaxy at the center of the stamp image are presented in Table 2.

\subsection{Radio and X-Ray Counterparts}

Six of the lens candidates have radio counterparts in the $1.4 \mathrm{GHz}$ VLA-COSMOS data (Schinnerer et al. 2007). The radio data cover

TABLE 3

Radio Information for the Strong Lenses

\begin{tabular}{|c|c|c|c|c|}
\hline $\begin{array}{l}\text { Name } \\
\text { (1) }\end{array}$ & $\begin{array}{c}\text { Flux } \\
(\mathrm{mJy}) \\
(2)\end{array}$ & $\begin{array}{l}\mathrm{S} / \mathrm{N} \\
(3)\end{array}$ & $\begin{array}{l}\text { Flag } \\
\text { (4) }\end{array}$ & $\begin{array}{l}\text { Comment } \\
\text { (5) }\end{array}$ \\
\hline 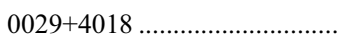 & 0.291 & 16.710 & 1 & Likely lensing galaxy counterpart \\
\hline 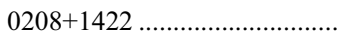 & 0.088 & 6.960 & 0 & Likely lensing galaxy counterpart \\
\hline 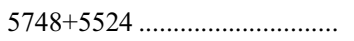 & 0.163 & 5.680 & 0 & Likely lensing galaxy counterpart \\
\hline 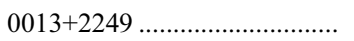 & 0.141 & 12.450 & 1 & Likely lensing galaxy counterpart \\
\hline 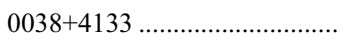 & 0.108 & 4.740 & 0 & Elongated, likely lensing galaxy counterpart \\
\hline 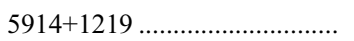 & 1.240 & 88.520 & 1 & Strong source, must be a quasar in the galaxy source or in the lens \\
\hline
\end{tabular}

Notes. - Col. (1): Lens candidate name. Col. (2): Total Flux in mJy. Col. (3): Signal-to-noise ratio of the detection. Col. (4): Resolved flag (1 = resolved radio source, $0=$ point source). Col. (5): Comment. 

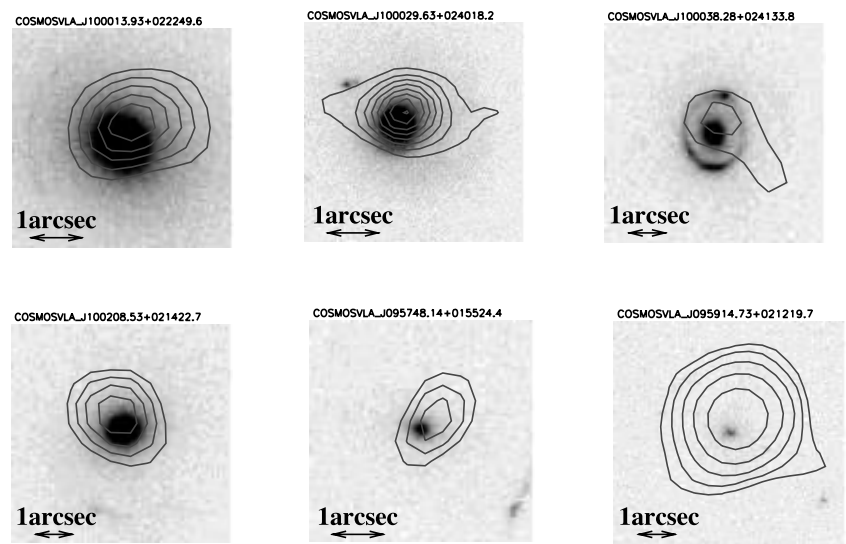

FIG. 7.- Radio contours on top of the ACS images. The name on top of each image refers to the radio source name in the VLA-COSMOS survey (Schinnerer et al. 2007) and are based on the radio source exact coordinates. The contours are defined as follows: $3,5,7,9,11,13,15$, and $17 \sigma$ for COSMOS 0013+2249 and $0029+4018 ; 3,4,5$, and $6 \sigma$ for COSMOS $0038+4133,0208+1422$, and $5748+$ $5524 ; 3,6,12,24$, and $48 \sigma$ for COSMOS 5914+1219. [See the electronic edition of the Supplement for a color version of this figure.]

the full COSMOS field to a depth of $\sim 10.5 \mu \mathrm{Jy}_{\text {beam }}{ }^{-1} \mathrm{rms}$ in the center of the field. All six sources lie above a $5 \sigma$ level in total flux, ranging from $77 \mu \mathrm{Jy}$ to $1.24 \mathrm{mJy}$ in total $1.4 \mathrm{GHz}$ flux density. The distance between optical and radio position is in all cases less than $0.5^{\prime \prime}$.

One of the lens candidates, COSMOS 0013+2249, which is also a radio emitter, shows associated X-ray point source emission from the $X M M$-Newton COSMOS survey (Brusa et al. 2007). It has $0.5-2 \mathrm{keV}$ X-ray emission of $3.74 \times 10^{-15} \pm 0.34 \times$ $10^{-16} \mathrm{erg}^{-1} \mathrm{~cm}^{-2} \mathrm{~s}^{-1}$, corresponding to an X-ray luminosity of $1.21 \times 10^{41} \mathrm{erg}^{-1} \mathrm{~s}^{-1}$ at the spectroscopic redshift of the lensing galaxy ( $z=0.347$; Trump et al. 2007). This X-ray emission as well as the optical spectrum from Magellan (Brusa et al. 2007) are consistent with the lensing galaxy being a radio galaxy with a central low-luminosity AGN. In Table 3 we list the radio counterpart properties and show VLA overlays on the ACS images in Figure 7.

\section{SIMPLE MASS MODELS FOR THE BEST SYSTEMS}

We want to estimate the Einstein radius of the lensing galaxies and determine if there are multiple images that are not detected, and/or if there is a need to take into account for the environment to model the system. We use the Lenstool code to make a parameterized mass model of the lens (Kneib et al. 1996, Jullo et al. 2007). The lensing galaxy is modeled by a singular isothermal ellipsoid (SIE). An external shear contribution is added when the SIE alone cannot reproduce the image configuration. The parameters optimized by the code are the coordinates, the orientation, the ellipticity and the velocity dispersion of the SIE, and the direction and amplitude of the external shear. The (unknown) redshift of the source is calculated assuming that the source is at twice the comoving distance of the lens. This assumption, while clearly incorrect in general, produces a lower limit for the estimated velocity dispersion of the lensing potential, since the efficiency of lensing is greatest for this configuration.

The Einstein radius is derived using the following equation:

$$
R_{\mathrm{E}}=4 \pi\left(\frac{\sigma_{v}}{c}\right)^{2} \frac{D_{\mathrm{ls}}}{D_{s}}
$$

where $D_{s}$ is the angular distance between the observer and the source and $D_{\mathrm{ls}}$ is the angular distance between the lensing galaxy and the source. The constraints used to optimize the mass model are the coordinates of the images. The multiplicity of the systems used in the model is given in Table 4. We first model the lens potential with a single SIE and let one or more of the SIE

TABLE 4

Morphological Parameters and Einstein Radil of the Lensing Galaxies for the Best Systems

\begin{tabular}{|c|c|c|c|c|c|c|c|c|}
\hline $\begin{array}{l}\text { Name } \\
(1)\end{array}$ & $\begin{array}{c}z_{l} \\
(2)\end{array}$ & $\begin{array}{l}z_{s} \\
(3)\end{array}$ & $\begin{array}{l}\chi^{2} \\
(4)\end{array}$ & $\begin{array}{c}\sigma_{v} \\
\left(\mathrm{~km} \mathrm{~s}^{-1}\right) \\
(5)\end{array}$ & $\begin{array}{l}R_{\mathrm{E}} \\
\left({ }^{\prime \prime}\right) \\
(6)\end{array}$ & $\begin{array}{l}\gamma, \theta_{\gamma} \\
(7)\end{array}$ & $\begin{array}{l}\text { More images? Comments } \\
\text { (8) }\end{array}$ & $\begin{array}{l}\text { Multiplicity } \\
\text { (9) }\end{array}$ \\
\hline $0018+3845 \ldots \ldots \ldots \ldots \ldots \ldots$ & 0.71 & 1.93 & 3.09 & 316.2 & 1.44 & $(0.18,-41.8)$ & No & Triple \\
\hline $0038+4133 \ldots \ldots \ldots \ldots \ldots \ldots$ & 0.89 & 2.70 & . & 240.0 & 0.83 & $(0.20,45.0)$ & No [1] & Ring \\
\hline 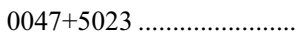 & 0.85 & 2.51 & 0.88 & 300.2 & 1.29 & $(0.18,80.0)$ & No & Triple \\
\hline 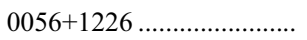 & 0.44 & 1.03 & 0.20 & 336.3 & 1.63 & No & No & Double \\
\hline 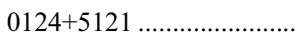 & 0.84 & 2.47 & $\ldots$ & 270.0 & 1.05 & No & No [1] & Ring \\
\hline 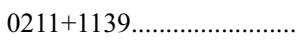 & 0.90 & 2.76 & 0.01 & 466.3 & 3.14 & No & No & 2xDouble \\
\hline 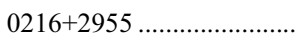 & 0.67 & 1.77 & 0.06 & 348.5 & 1.75 & No & No & Double \\
\hline $0227+0451 \ldots \ldots \ldots \ldots \ldots \ldots \ldots$ & 0.89 & 2.70 & 0.01 & 369.8 & 1.97 & No & No [2] & Double \\
\hline $5857+5949$ & 0.39 & 0.89 & 0.94 & 361.5 & 1.88 & No & Yes [3] & Double \\
\hline 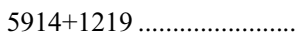 & 1.05 & 3.57 & 4.70 & 442.8 & 2.82 & $(0.18,-31.7)$ & No [2] & Quad \\
\hline
\end{tabular}

Notes.-Col. (1): Name. Cols. (2) and (3): Lens and source redshift used for the modeling. Col. (4): $\chi^{2}$ of the fit between the best mass model and the data. Cols. (5) and (6): Velocity dispersion and Einstein radius of the lens. Col. (7): Value and orientation of the shear if used in the modeling. Col. (8): Do more images appear during the modeling? Yes or no. The numbers refer to the following comments: [1] The mass model is made using a source aligned with the lens center, and we scale $\sigma_{v}$ to fit the ring size. [2] The angular distance between the images and the velocity dispersion of the lens makes us think that the lensing galaxy is associated with a galaxy group or cluster. Some close-by galaxies to the systems are also seen and can generate the external shear. [3] Even the best-fit models predict two more images not visible in the ACS data. [4] The light profile of the lensing galaxy at the Einstein radius has a different orientation $\left(\theta=59.0^{\circ}\right)$ than the light profile at the effective radius $\left(\theta=26.6^{\circ}\right)$. The mass model reproduces the configuration of the lensed object if the first orientation is considered for the lens.. Col. (9): Multiplicity of the images used for the mass modeling. 


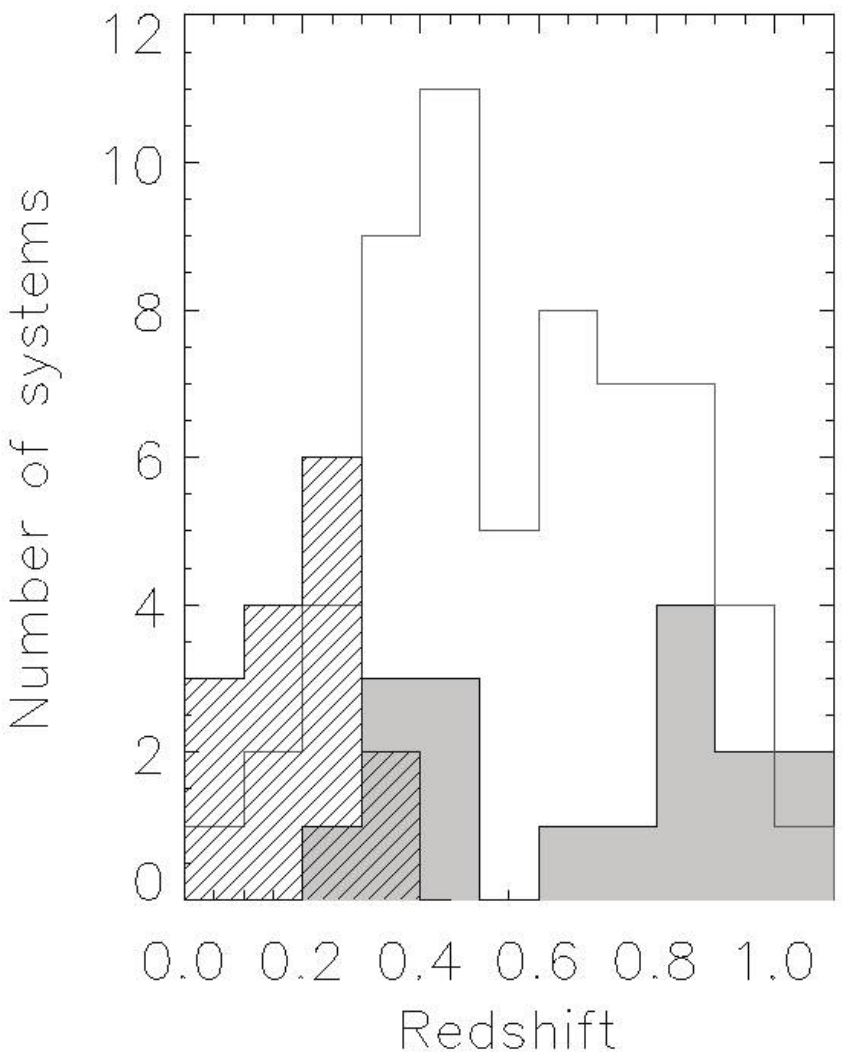

FIG. 8.- Spectroscopic redshift distribution of the lensing galaxies in the SLACS survey (hatched histogram) and in the CASTLES database (solid histogram), compared to the photometric redshift distribution of the best systems in the COSMOS survey (gray shaded histogram).

parameters vary freely. The model uncertainties are typically \pm 0.1 for the ellipticity and $\pm 10^{\circ}$ for the orientation, reflecting a possible misalignment between the mass and the light (Kochanek 2002). If necessary, and if there are enough constraints, an external shear contribution can be added. For the ringlike objects, we have optimized the velocity dispersion of the SIE by hand in order to retrieve a perfect Einstein ring. For the purposes of this calculation, we assume that the source, the lens, and the observer are perfectly aligned. Thus, we have not given any $\chi^{2}$ value for these cases in Table 4. The system COSMOS $0013+2249$ is not included in the table, as it possesses a single visible elongated and curved arc, providing too few constraints to fit a mass model.

In Table 4 we display the velocity dispersion and the external shear parameters corresponding to the best fits. Additional details are given in comments in the caption when the result of the fitting procedure is not straightforward.

\section{ANALYSIS OF THE SAMPLE AND COMPARISON TO OTHER STRONG LENSING SURVEYS}

In this section we compare the physical parameters of the foreground in the COSMOS strong lens sample with independent galaxy-galaxy strong lensing catalogs from the SLACS survey (Treu et al. 2005; Bolton et al. 2006) and the CASTLES database $^{16}$ (Muñoz et al. 1998). Although the SLACS and CASTLES samples were selected very differently from the COSMOS strong lensing sample, they also have HST imaging. Thus, a rough comparison in terms of the galaxy lensing properties is warranted, at least for the subsample of best systems.

\footnotetext{
16 See http://www.cfa.harvard.edu/castles/.
}

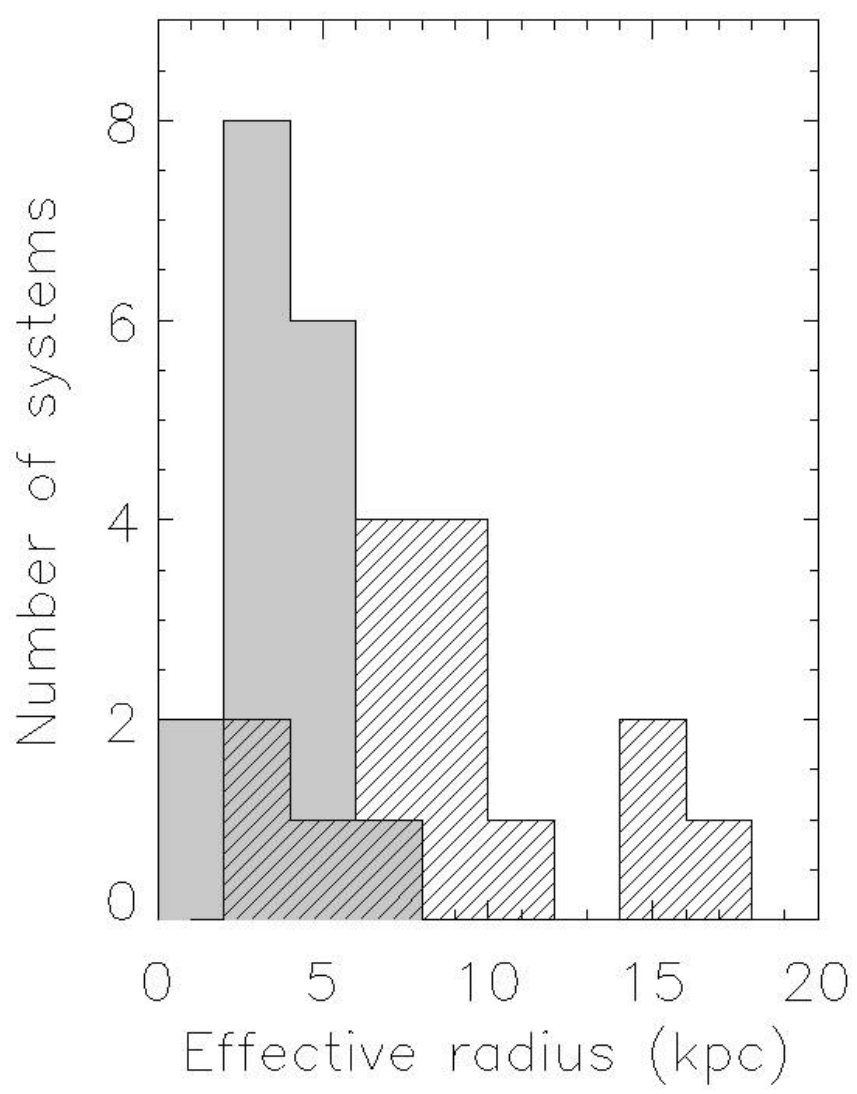

FIG. 9.-Effective radii of strong lenses in the SLACS survey (hatched histogram) and the best systems from the COSMOS survey (gray shaded histogram).

\subsection{Redshift and Effective Radius}

Photometric redshifts have been measured by Mobasher et al. (2007) for most of the COSMOS strong lensing galaxy candidates (see Tables 1 and 2). We first compare the redshift distribution of the lensing galaxy candidates found in the COSMOS field ( 17 best candidates with a photometric redshift) to the lensing galaxies in the CASTLES database (90 strong galaxy-galaxy lenses, lens redshift known for 59 of them) and in the SLACS survey (19 strong galaxy-galaxy lenses; Fig. 8). The (photometric) redshift distribution of the COSMOS sample of strong lenses extends to significantly higher redshifts than the spectroscopic redshift distribution of galaxies in the SLACS sample, which is intrinsically limited due to the SDSS depth, but is similar to the spectroscopic redshift distribution of galaxies in the CASTLES sample.

The effective radii and arc radius of the lensing galaxies (in kiloparsecs) are simply obtained by converting the effective radii in angular units to physical distances, via the photometric redshift of the lensing galaxy. The SLACS sample includes lenses with larger effective lensing radii than the COSMOS sample (see Fig. 9). This is probably an observational bias, as (for practical reasons) we visually inspect the lens candidates in a fixed $10^{\prime \prime} \times$ $10^{\prime \prime}$ box and therefore miss any galaxies that would generate arcs at larger radii.

\subsection{The Absolute Magnitude}

The absolute magnitudes of the SLACS lensing galaxies are available in Treu et al. (2006). The mean value of their $V$-band magnitude is $\left\langle M_{V}\right\rangle=-22.71 \pm_{2.46}^{1.23}$. The absolute $V$-band magnitudes of the lensing galaxy candidates of our best systems sample have a mean value of $\left\langle M_{V}\right\rangle=-21.92 \pm_{1.23}^{1.78}$. Thus, the COSMOS 


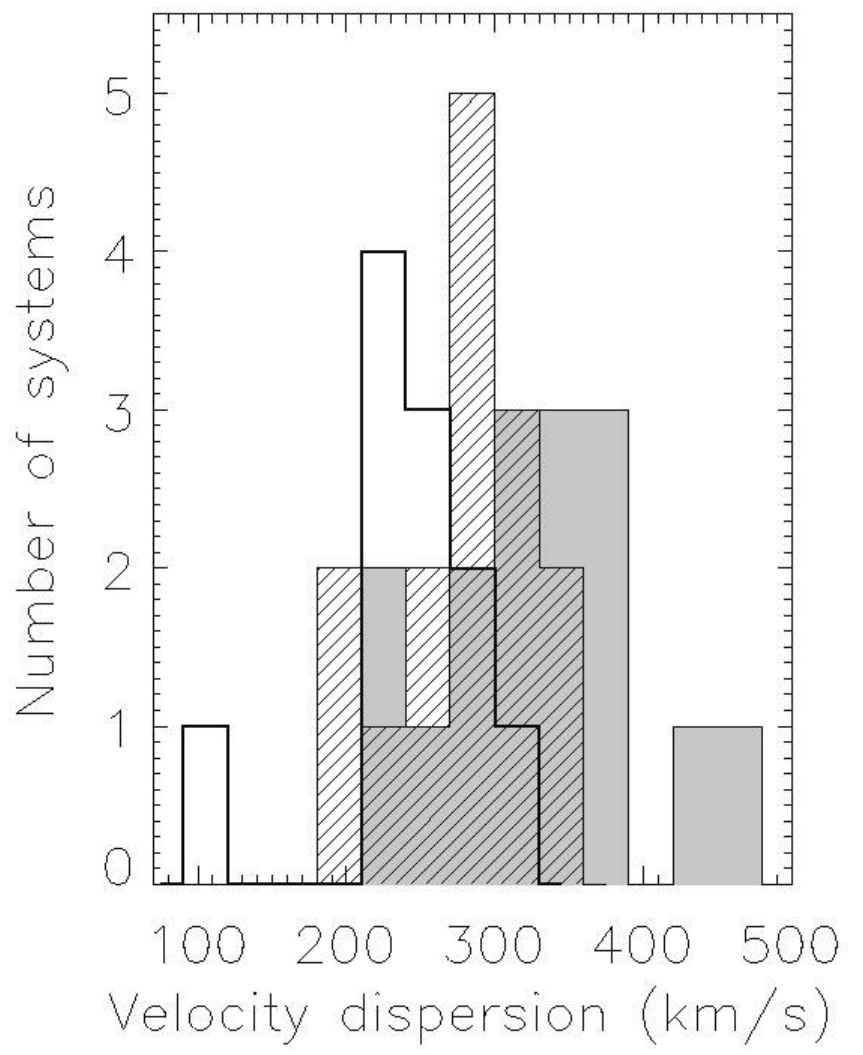

FIG. 10.- Velocity dispersions of strong lenses in the SLACS survey (hatched histogram) and in the CASTLES database (solid histogram) compared to the velocity dispersions of the best systems in the COSMOS sample (gray shaded histogram).

sample reaches slightly fainter magnitudes than the SLACS sample, despite the fact that it extends to higher lens redshifts.

\subsection{The Velocity Dispersion}

The velocity dispersions of the lensing galaxies of the COSMOS sample are derived from simple mass models presented in $\S 5$. They are displayed in Table 4 . We have compared the velocity dispersions of the lensing galaxy candidates of the best systems to the velocity dispersions of the lensing galaxies in the SLACS and in the CASTLES samples (also measured using isothermal mass potentials; Fig. 10). The lensing galaxies in the COSMOS sample have a slightly higher velocity dispersion than the lensing galaxies in SLACS and in CASTLES. The difference with the SLACS sample can be due to the fact that we are probing in mean larger arc radius (therefore larger Einstein radius). In addition, we have estimated the source redshift from the lensing galaxy photometric redshifts, that have an uncertainty. This can lead in some cases to wrong estimations of the velocity dispersion of the main deflector. In particular, we note that two systems have a high-velocity dispersion $\left(>400 \mathrm{~km} \mathrm{~s}^{-1}\right)$ : COSMOS $0211+1139$ and COSMOS 5914+1219. If real, these values suggest that these galaxies are in the center of a group, and that we are measuring here both the galaxy mass and the group mass.

\subsection{The Einstein Radius}

The Einstein radius is directly derived from the velocity dispersion of the lensing galaxy; it is therefore another mass estimator for the lens potential. It has the advantage of being directly comparable to the observable arc radius. The value of the Einstein

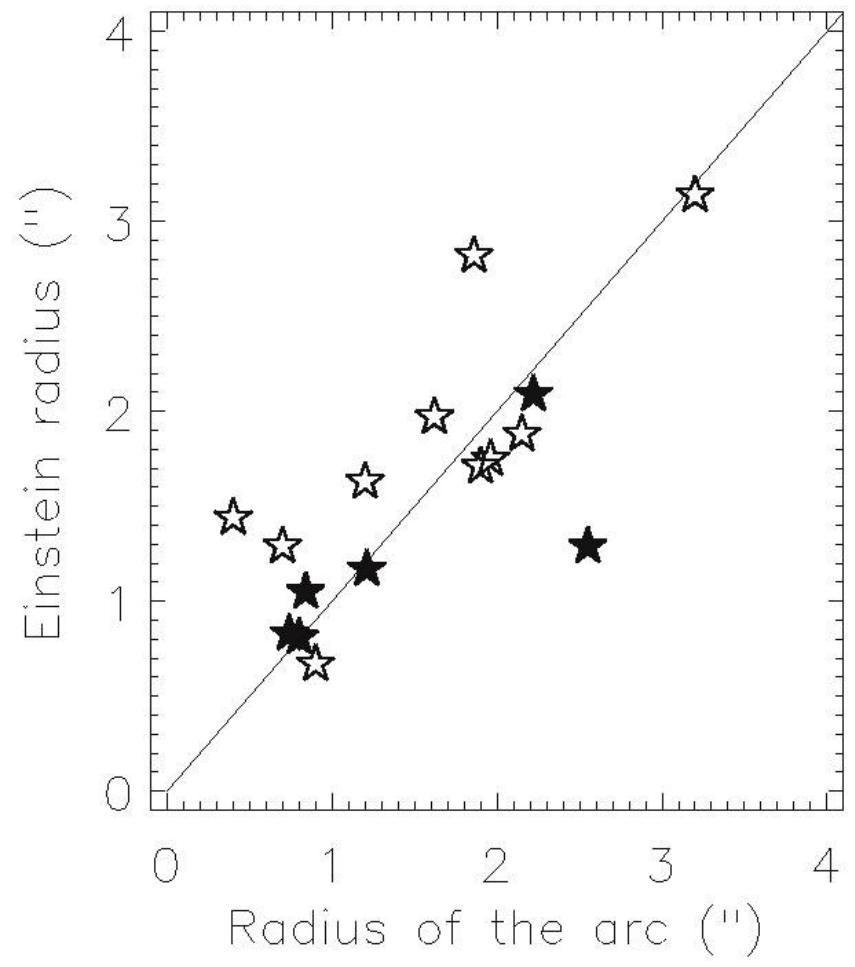

FIG. 11.-Arc radius vs. the Einstein radius of the best systems. The black stars show the ringlike candidates. The solid line traces $R_{\mathrm{E}}=r_{\text {arc }}$.

radii of the lensing galaxies $R_{\mathrm{E}}$ are given in Table 4 . For the best systems, the Einstein radius is expected to be similar to the arc radius, while an equality between these two values is expected in case of perfect alignment between the observer, the source, and the lens (Einstein ring). The plot in Figure 11 shows that the two radii are indeed similar for most of the observed systems. Nevertheless, we observe some exceptions; some systems have a large Einstein radius in comparison to their arc radius: COSMOS $0018+3845$ $\left(r_{\text {arc }}=0.40^{\prime \prime}, R_{\mathrm{E}}=1.44^{\prime \prime}\right), 0047+5023\left(r_{\mathrm{arc}}=0.70^{\prime \prime}, R_{\mathrm{E}}=\right.$ $\left.1.29^{\prime \prime}\right)$, and $5914+1219\left(r_{\mathrm{arc}}=1.86^{\prime \prime}, R_{\mathrm{E}}=2.82^{\prime \prime}\right)$. For the three systems, there is a strong external shear added to the SIE. This may indicate the presence of a massive structure in the direction of the source or in its vicinity that could have stretched the angular separation between the images and/or biased strongly the estimation of the velocity dispersion and hence the estimation of the Einstein radius of the lensing galaxy.

\section{DISCUSSION AND CONCLUSION}

In this paper we present 67 new strong galaxy-galaxy lensing candidates found by visual inspection of the COSMOS field. From a catalog of 278,526 galaxies with photometric redshifts (Capak et al. 2007), we have selected a subcatalog of 9452 bright galaxies $\left(M_{V}<-20 \mathrm{mag}\right)$, at intermediate redshift $\left(0.2 \leq z_{\text {phot }} \leq\right.$ $1.0)$ and spectrally early type. The selection criteria for this subcatalog were mainly motivated by the purpose of reducing the sample of galaxies to be visually inspected. From the visual inspection of ACS $I_{\mathrm{F} 814 \mathrm{~W}}$-band stamp images of $10^{\prime \prime} \times 10^{\prime \prime}$, we have built a sample of 337 possible lenses with sizes up to $r_{\text {arc }} \sim 5^{\prime \prime}$. This number is reduced to 67 strong galaxy-galaxy lensing candidates after a final cut based on an inspection of the pseudocolor images, and the fit and subtraction of the lensing galaxy luminosity profile. There are 47 candidates with a single arclet and 20 candidates with multiple images of a background source or 
with a large curved arc, called the best systems in this paper. We have produced a simple mass model (SIE+shear) for each of the best candidates. In a few cases, we have also been able to predict the existence and direction of an external shear contribution. Comparing the velocity dispersions to those of lenses in the SLACS and CASTLES samples, it is apparent that we are probing a slightly higher range of velocity dispersion. Nevertheless, massive objects in the immediate vicinity of the COSMOS lenses, and the rough assumption concerning the source redshift, can explain this result. We have inferred the Einstein radius for the lensing galaxies considering that they are isothermal spheres and compared these values with the arc radius. The derived Einstein radii agree well with the observed arc radii, making us confident that the sample consists mainly of genuine lens systems.

The "visual inspection" approach is long and tedious and does not ensure a full understanding of the completeness of the strong lenses sample. Nevertheless, our meticulously obtained large sample of strong lenses, even if incomplete, provides a valuable catalog to help develop, test, and improve automatic strong lens-finding algorithms for similar data sets. Such work is currently in progress (Cabanac et al. 2007; P. Marshall et al. 2008, in preparation; Seidel \& Bartlemann 2007). Also, in future work (C. Faure et al. 2008, in preparation) the direct study of the spatial correlation between large-scale structure in the COSMOS field (Scoville et al. 2007; Massey et al. 2007) and the strong lenses discovered here will allow us to measure the impact of the total, projected mass distribution along the line of sight to a given strong lens, as recently investigated for three strong lenses in the AEGIS survey (Moustakas et al. 2007). A spectroscopic follow-up of the lens candidates is in progress at the VLT using the FORS1Multi-Object Spectroscopy capability. This will allow us to measure precisely the redshift of both the lens and the source and to explore the close neighborhood of the lens, thus allowing a definite measurement of the mass of each strong lensing galaxy.

Finally, this study gives a lower limit on the number of strong lenses expected in future deep space surveys such as the proposed $J D E M / S N A P$ multicolor weak-lensing survey. Indeed, we expect to find at least 10 strong lenses per square degree. Thus, for a $10,000 \mathrm{deg}^{2}$ survey, we should discover about 100,000 strong lenses, thus allowing a high-precision statistical analysis of the mass properties of galaxies and its evolution over time.

The HST COSMOS Treasury program was supported through NASA grant HST-GO-09822. We wish to thank Tony Roman, Denise Taylor, and David Soderblom for their assistance in the planning and scheduling of the extensive COSMOS observations. We gratefully acknowledge the contributions of the entire COSMOS collaboration consisting of more than 70 scientists. More information on the COSMOS survey is available at http://www .astro.caltech.edu/cosmos. Based on observations with the NASA/ ESA Hubble Space Telescope, obtained at the Space Telescope Science Institute, which is operated by the Association of Universities for Research in Astronomy (AURA) Inc., under NASA contract NAS 05-26555; also based on data collected at the Subaru Telescope, which is operated by the National Astronomical Observatory of Japan; the European Southern Observatory under Large Program 175.A-0839, Chile; Kitt Peak National Observatory, Cerro Tololo Inter-American Observatory, and the National Optical Astronomy Observatory, which are operated by AURA, Inc., under cooperative agreement with the National Science Foundation. Based on observations obtained with MegaPrime/MegaCam, a joint project of CFHT and CEA/DAPNIA, at the Canada-FranceHawaii Telescope which is operated by the National Research Council of Canada, the Institute National des Sciences de l'Univers of the Centre National de la Recherche Scientifique of France, and the University of Hawaii. It is a pleasure to acknowledge the excellent services provided by the NASA IPAC/IRSA staff(Anastasia Laity, Anastasia Alexov, Bruce Berriman, and John Good) in providing online archive and server capabilities for the COSMOS data sets. C. F. acknowledges support from the European Community's Sixth Framework Marie Curie Research Training Network Programme, contract MRTN-CT-2004-505183 “ANGLES.” J. P. K. acknowledgees support from CNRS and Caltech. G. C. is partially supported by the Cordis ERG proposal 029159. We thank the referee for useful remarks and J. Duke for correcting the draft.

Facilities: HST (ACS), Subaru (Suprime). CFHT (Megacam).
Barkana, R. 1997, ApJ, 489, 21

Bolton, A. S., Burles, S., Koopmans, L. V. E., Treu, T., \& Moustakas, L. A. 2006, ApJ, 638, 703

Boulade, O., et al. 2003, Proc. SPIE, 4841, 72

Browne, I. W. A., Wilkinson, P. N., Patnaik, A. R., \& Wrobel, J. M. 1998, MNRAS, 293, 257

Brusa, M., et al. 2007, ApJS, 172, 353

Burud, I., et al. 2000, ApJ, 544, 117

2002a, A\&A, 383, 71

2002b, A\&A, 391, 481

Cabanac, R. A., et al. 2007, A\&A, 461, 813

Capak, P., et al. 2007, ApJS, 172, 99

Chae, K.-H. 2007, ApJ, 658, L71

Chae, K.-H., Mao, S., \& Kang, X. 2006, MNRAS, 373, 1369

Cohen, A. S., Hewitt, J. N., Moore, C. B., \& Haarsma, D. B. 2000, ApJ, 545, 578

Dickinson, M., et al. 2001, BAAS, 33, 820

Estrada, J., et al. 2007, ApJ, 660, 1176

Fassnacht, C. D., Moustakas, L. A., Casertano, S., Ferguson, H. C., Lucas, R. A., \& Park, Y. 2004, ApJ, 600, L155

Fassnacht, C. D., Xanthopoulos, E., Koopmans, L. V. E., \& Rusin, D. 2002, ApJ, 581, 823

Finoguenov, A., et al. 2007, ApJS, 172, 182

Fohlmeister, J., et al. 2007, ApJ, 662, 62 \&Ponman, T. J. 1999, MNRAS, 309, 623

Fukugita, M., \& Turner, E. L. 1991, MNRAS, 253, 99

Gladders, M. D., Yee, H. K. C., McCarthy, P. J., Barrientos, L. F., Hoekstra, H.,

Hall, P. B., \& Ellingson, E. 2002, BAAS, 34, 1209

Griffiths, R. E., et al. 1994, BAAS, 26, 877

\section{REFERENCES}

Guzik, J., \& Seljak, U. 2002, MNRAS, 335, 311

Hennawi, J. F., et al. 2008, AJ, 135, 664

Hilbert, S., White, S. D. M., Hartlap, J., \& Schneider, P. 2007, MNRAS, 382, 121

Hjorth, J., et al. 2002, ApJ, 572, L11

Ilbert, O., et al. 2006, A\&A, 457, 841

Jackson, N., et al. 1995, MNRAS, 274, L25

Jakobsson, P., Hjorth, J., Burud, I., Letawe, G., Lidman, C., \& Courbin, F. 2005, A\&A, 431, 103

Jullo, E., Kneib, J.-P., Limousin, M., Elíasdóttir, Á., Marshall, P., \& Verdugo, T. 2007, New J. Phys., 9, 447

Kneib, J.-P., Ellis, R. S., Smail, I., Couch, W. J., \& Sharples, R. M. 1996, ApJ, 471,643

Kochanek, C. S. 1996, Astrophysical Applications of Gravitational Lensing, 173,177

2002, in The Shapes of Galaxies and Their Dark Halos, ed. Priyamvada Natarajan (Singapore: World Scientific), 62

Kochanek, C. S., Morgan, N. D., Falco, E. E., McLeod, B. A., Winn, J. N., Dembicky, J., \& Ketzeback, B. 2006, ApJ, 640, 47

Koekemoer, A. M., et al. 2007, ApJS, 172, 196

Leauthaud, A., et al. 2007, ApJS, 172, 219

Lilly, S. J., et al. 2007, ApJS, 172, 70

Mandelbaum, R., Seljak, U., Kauffmann, G., Hirata, C. M., \& Brinkmann, J. 2006, MNRAS, 368, 715

Marleau, F. R., \& Simard, L. 1998, ApJ, 507, 585

Marshall, P. J., Moustakas, L. A., Hogg, D. W., Bradac, M., Fassnacht, C. D., \& Blandford, R. D. 2005, BAAS, 37, 1498 
Massey, R., et al. 2007, Nature, 445, 286

Miralda-Escude, J., \& Lehar, J. 1992, MNRAS, 259, 31P

Miyazaki, S., et al. 2002, PASJ, 54, 833

Mobasher, B., et al. 2007, ApJS, 172, 117

Moeller, O., Kitzbichler, M., \& Natarajan, P. 2007, MNRAS, 379, 1195

Moustakas, L. A., et al. 2007, ApJ, 660, L31

Muñoz, J. A., et al. 1998, Ap\&SS, 263, 51

Myers, S. T., et al. 1995, ApJ, 447, L5

Ofek, E. O., \& Maoz, D. 2003, ApJ, 594, 101

Patnaik, A. R., Browne, I. W. A., Wilkinson, P. N., \& Wrobel, J. M. 1992, MNRAS, 254, 655

Peng, C. Y., Falco, E. E., Lehar, J., Impey, C. D., Kochanek, C. S., McLeod, B. A., \& Rix, H.-W. 1997, BAAS, 29, 1348

Ratnatunga, K. U., Griffiths, R. E., \& Ostrander, E. J. 1999, AJ, 117, 2010

Read, J. I., Saha, P., \& Maccio, A. V. 2007, ApJ, 667, 645

Refsdal, S. 1964, MNRAS, 128, 307
Rusin, D., \& Ma, C.-P. 2001, ApJ, 549, L33

Scarpine, V., et al. 2006, BAAS, 209, 215.07

Scoville, N. Z., et al. 2007, ApJS, 172, 150

Schinnerer, E., et al. 2007, ApJS, 172, 46

Seidel, G., \& Bartelmann, M. 2007, A\&A, 472, 341

Simard, L. 1998, in ASP Conf. Ser. 145, Astronomical Data Analysis Software and Systems VII, ed. R. Albrecht, R. N. Hook, \& H. A. Bushouse (San Francisco: ASP), 108

Treu, T., Koopmans, L. V. E., Bolton, A. S., Burles, S., \& Moustakas, L. A. 2005, BAAS, 37, 1498

2006, ApJ, 640, 662

Trump, J. R., et al. 2007, 172, 383

Vuissoz, C., et al. 2007, A\&A, 464, 845

Wambsganss, J., Bode, P., \& Ostriker, J. P. 2005, ApJ, 635, L1

Wisotzki, L., Koehler, T., Groote, D., \& Reimers, D. 1996, A\&AS, 115, 227 